\title{
On the problem of a consistent description of kinetic and hydrodynamic processes in dense gases and liquids
}

\author{
B.B. Markiv, I.P. Omelyan, M.V. Tokarchuk \\ Institute for Condensed Matter Physics of the National Academy of Sciences of Ukraine, \\ 1 Svientsitskii Str., 79011 Lviv, Ukraine
}

Received March 19, 2010, in final April 8, 2010

For a consistent description of kinetic and hydrodynamic processes in dense gases and liquids the generalized non-Markovian equations for the nonequilibrium one-particle distribution function and potential part of the averaged enthalpy density are obtained. The inner structure of the generalized transport kernels for these equations is established. It is shown that the collision integral of the kinetic equation has the Fokker-Planck form with the generalized friction coefficient in momentum space. It also contains contributions from the generalized diffusion coefficient and dissipative processes connected with the potential part of the enthalpy density.

Key words: kinetics, hydrodynamics, kinetic equations, memory functions, time correlation functions

PACS: $05.20 . D d, 05.60 .+w, 52.25 . F i, 82.20 . \mathrm{M}$

\section{Introduction}

A number of investigations [1 12] was devoted to the problem of constructing a consistent description of kinetic and hydrodynamic processes in dense gases, liquids, and plasma. The paper by D.N. Zubarev and V.G. Morozov [3] is a fundamental one in kinetic theory of dense gases and liquids. In that paper the formulation of a new boundary condition to the BBGKY hierarchy for nonequilibrium distribution functions which takes into account correlations connected with local conservation laws was given. In a pair collisions approximation this modification of Bogolyubov's correlation weakening condition allows one to consistently derive the Enskog kinetic equations [3] for neutral and one-component charged hard sphere systems. In the paper by Yu.L. Klimontovich [6] the necessity and possibility of a consistent description of kinetic and hydrodynamic processes in gases for values of the Knudsen number of order of unity were substantiated. Herewith, the relation $\left(\tau_{\mathrm{ph}}^{G}\right)_{\min } \sim L_{\min }^{2} / D \sim \tau_{\mathrm{ph}}$ takes place, where $\tau_{\mathrm{ph}}$ denotes the physically infinitesimal time interval during which one particle suffers a collision from a collection with $N_{\mathrm{ph}}=n V_{\mathrm{ph}}=\frac{N}{V} V_{\mathrm{ph}}$ particles in small volume $V_{\mathrm{ph}}$ with physically infinitesimal length scale $l_{\mathrm{ph}}$, where $D$ is the kinetic coefficient, $\left(\tau_{\mathrm{ph}}^{G}\right)_{\min } \sim\left(l_{\mathrm{ph}}^{G}\right)_{\min }^{2} / D$ (diffusive relation) is the minimal hydrodynamic time typical of diffusive process, $L_{\min }$ is the minimal length, on which the diffusive relation is still correct. The latter is lesser than the free motion path $l$ but larger with respect to the scale $l_{\mathrm{ph}}$ corresponding to the kinetic description. Basing on these arguments, the generalized kinetic equation for the description of nonequilibrium processes at arbitrary Knudsen numbers was proposed. In particular, the collision integral of this equation contains the diffusion coefficient in velocity space. A regular perturbation theory can be implemented for solving such an equation. Using the ideas of paper [3,, 4$]$ the generalized transport equations for the hydrodynamic variables such as number of particles, momentum and total energy densities were obtained in [7, 9] by means of nonequilibrium statistical operator method. Such equations are consistent with the kinetic equation for the nonequilibrium one-particle distribution function. They allow one to study the time correlation functions describing the dynamics of a liquid system in states close to equilibrium. These results are equivalent to those obtained in the investigations of [1, 2] based on the Mori projection operator method. An important 
common problem of these investigations consists in the calculation of the generalized transport kernels (memory functions). The latter determine the collision integrals for various models $\mid 1$, 1324] as well as the generalized transport coefficients such as viscosity and heat conductivity [1, 3, 4]. This problem is particularly significant in the region of short-ranged interactions. The reason is that at small distances according to Klimontovich one obtains the changes not only for the spatiallytemporal characteristics of particles dynamics but also for momenta during the collisions of particles inside the physical volume $V_{\mathrm{ph}}$ at times $\tau_{\mathrm{ph}}$. The importance of taking into account the kinetic processes connected with irreversible collision processes at the scale of short-ranged interparticle interactions was pointed out in [25], for instance. The short-wavelength collective modes in liquids were investigated therein on the basis of a linearized kinetic equation of the revised Enskog theory for the model of hard spheres.

In section 2 of this paper, a nonequilibrium statistical operator is derived within a consistent description of kinetic and hydrodynamic processes for the system of classical interacting particles. In section 3, on the basis of this operator, the set of transport equations for the nonequilibrium oneparticle distribution function and the potential part of the averaged enthalpy density is obtained in the case of weakly nonequilibrium processes. In the fourth section, the inner structure of generalized transport kernels of a consistent description of kinetic and hydrodynamic processes is analyzed. It is shown that they can be expressed in terms of time correlation functions corresponding to the basic set of dynamical variables, phase density of microscopic particle distribution $\hat{n}(x)$, potential part of the enthalpy density $\hat{h}_{\vec{k}}^{\text {int }}(\vec{r})$ and generalized transport kernels describing diffusive, viscous and heat-conduction processes.

\section{Nonequilibrium statistical operator for a consistent description of ki- netics and hydrodynamics of system}

In dense gases and liquids there is no small parameter and the characteristic time of interparticle correlations is comparable with those for the one-particle distribution function. This means that during the particle collision process the many-particles correlations related to local mass, momentum and energy conservation laws, underlying the hydrodynamic description of a system, cannot be neglected. In this connection the local conservation laws impose some restrictions on the kinetic processes. Their role is especially important at high densities, when the interaction between a separate group of particles and other ones cannot be neglected. This indicates a close connection between the kinetic and hydrodynamic processes in dense gases and liquids [3, 4]. Therefore, to obtain the kinetic equations for these systems it is quite natural to choose the reduced description of nonequilibrium states and in such a way to automatically take into account a proper dynamics of conserved quantities. For this purpose we can include densities of hydrodynamic variables together with $f_{1}(x ; t)$ into the set of reduced-description parameters at the very beginning. The phase functions

$$
\begin{aligned}
& \hat{\rho}(\vec{r})=m \int \mathrm{d} \vec{p} \hat{n}_{1}(x)=m \hat{n}(\vec{r}), \quad \hat{\vec{\jmath}}(\vec{r})=m \int \mathrm{d} \vec{p} \vec{p} \hat{n}_{1}(x), \\
& \hat{\varepsilon}(\vec{r})=\int \mathrm{d} \vec{p} \frac{p^{2}}{2 m} \hat{n}_{1}(x)+\frac{1}{2} \int \mathrm{d} \vec{p} \int \mathrm{d} \overrightarrow{p^{\prime}} \int \mathrm{d} \overrightarrow{r^{\prime}} \Phi\left(\left|\vec{r}-\overrightarrow{r^{\prime}}\right|\right) \hat{n}_{2}\left(x, x^{\prime}\right),
\end{aligned}
$$

correspond to the densities of hydrodynamic quantities $\rho(\vec{r} ; t), \vec{\jmath}(\vec{r} ; t)$ and $\varepsilon(\vec{r} ; t)$. Here

$$
\hat{n}_{1}(x)=\sum_{l=1}^{N} \delta\left(x-x_{l}\right)=\sum_{l=1}^{N} \delta\left(\vec{p}-\vec{p}_{l}\right) \delta\left(\vec{r}-\vec{r}_{l}\right), \quad \hat{n}_{2}\left(x, x^{\prime}\right)=\sum_{l \neq j=1}^{N} \delta\left(x-x_{l}\right) \delta\left(x^{\prime}-x_{l}\right),
$$

are the phase densities of the microscopic distribution of particles, $x_{j}=\left\{\vec{p}_{j}, \vec{r}_{j}\right\}$ the phase variables of $j$-particle, $\Phi\left(r_{l j}\right)$ the interaction energy of two particles, $\vec{p}_{j}$ the $j$-th particle momentum, $m$ is its mass, and $r_{l j}=\left|\vec{r}_{l}-\vec{r}_{j}\right|$ denote the distance between a pair of interacting particles.

From expressions (11) we can see an important role of potential energy of interaction. Contrary to $\rho(\vec{r} ; t)=\langle\hat{\rho}(\vec{r})\rangle^{t}$ and $\vec{\jmath}(\vec{r} ; t)=\langle\hat{\vec{\jmath}}(\vec{r})\rangle^{t}$, the nonequilibrium average $\varepsilon(\vec{r} ; t)=\langle\hat{\varepsilon}(\vec{r})\rangle^{t}$ cannot 
be expressed only via one-particle distribution function $f_{1}(x ; t)=\left\langle\hat{n}_{1}(x)\right\rangle^{t}$. Indeed, as it follows from (11), the potential part of energy $\varepsilon_{\text {int }}(\vec{r} ; t)=\left\langle\hat{\varepsilon}_{\text {int }}(\vec{r})\right\rangle^{t}$ is calculated by means of two-particle distribution function $f_{2}\left(x, x^{\prime} ; t\right)=\left\langle\hat{n}_{2}\left(x, x^{\prime}\right)\right\rangle^{t}$, where

$$
\hat{\varepsilon}_{\text {int }}(\vec{r})=\frac{1}{2} \int \mathrm{d} \vec{p} \int \mathrm{d} \overrightarrow{p^{\prime}} \int \mathrm{d} \overrightarrow{r^{\prime}} \Phi\left(\left|\vec{r}-\overrightarrow{r^{\prime}}\right|\right) \hat{n}_{2}\left(x, x^{\prime}\right)
$$

is the density of potential energy. Obviously, if $f_{1}(x ; t)$ is chosen as one of a parameters of the reduced description, then the average value of the interaction energy density can be taken as an additional independent parameter. $\langle\ldots\rangle^{t}=\int \mathrm{d} \Gamma_{N} \cdots \varrho\left(x^{N} ; t\right)$ denotes the nonequilibrium average calculated by means of nonequilibrium distribution function $\varrho\left(x^{N} ; t\right)$. The latter satisfies the Liouville equation

$$
\frac{\partial}{\partial t} \varrho\left(x^{N} ; t\right)+\mathrm{i} L_{N} \varrho\left(x^{N} ; t\right)=0,
$$

where

$$
\mathrm{i} L_{N}=\sum_{l=1}^{N} \frac{\vec{p}_{l}}{m} \cdot \frac{\partial}{\partial \vec{r}_{l}}-\frac{1}{2} \sum_{l \neq j=1}^{N} \frac{\partial}{\partial \vec{r}_{l}} \Phi\left(r_{l j}\right)\left(\frac{\partial}{\partial \vec{p}_{l}}-\frac{\partial}{\partial \vec{p}_{j}}\right)
$$

is the Liouville operator of the system. The function $\varrho\left(x^{N} ; t\right)$ is symmetric with respect to inversion of phase variables of any pair of particles $\left(x_{l} \leftrightarrow x_{j}\right)$ and satisfies the normalization condition $\int \mathrm{d} \Gamma_{N} \varrho\left(x^{N} ; t\right)=1, \mathrm{~d} \Gamma=(\mathrm{d} x)^{N} / N !, \mathrm{d} x=\mathrm{d} \vec{p} \mathrm{~d} \vec{r}$.

In order to solve the Liouville equation (4) we will use the Zubarev nonequilibrium statistical operator method [10, 11]. Within its framework we will be looking for solutions of the equation (44), which are independent of the initial conditions. The solutions will depend on time only explicitly, i.e. through the observable quantities selected for a reduced description of nonequilibrium states of the system. When the basic parameters of a reduced description are chosen, the solution $\varrho\left(x^{N} ; t\right)$ can be presented using the nonequilibrium statistical operator method in the following general form $[10,11]$ :

$$
\varrho\left(x^{N} ; t\right)=\varrho_{q}\left(x^{N} ; t\right)-\int_{-\infty}^{t} \mathrm{e}^{\varepsilon\left(t^{\prime}-t\right)} T\left(t, t^{\prime}\right)\left(1-P_{q}\left(t^{\prime}\right)\right) \mathrm{i} L_{N} \varrho_{q}\left(x^{N} ; t^{\prime}\right) \mathrm{d} t^{\prime},
$$

where $T\left(t, t^{\prime}\right)=\exp _{+}\left\{-\int_{t^{\prime}}^{t}\left(1-P_{q}\left(t^{\prime}\right)\right) \mathrm{i} L_{N} \mathrm{~d} t^{\prime}\right\}$ is the evolution operator containing projection; $\exp _{+}$is the ordered exponential. $P_{q}\left(t^{\prime}\right)$ is the generalized Kawasaki-Gunton projection operator whose structure depends on the form of quasiequilibrium distribution function $\varrho_{q}\left(x^{N} ; t\right)$. The latter is determined according to Gibbs and corresponds to a maximum of the entropy of the system at fixed parameters of the reduced description with taking into account the normalization condition. In our case, the basic parameters are the nonequilibrium one-particle distribution function $f_{1}(x ; t)$ and the averaged density of the interaction energy of the system $\left\langle\hat{\varepsilon}_{\text {int }}(\vec{r})\right\rangle^{t}$. Then, the quasiequilibrium distribution function $\varrho_{q}\left(x^{N} ; t\right)$ providing a maximum for the entropy of the system at fixed averaged values $f_{1}(x ; t)$ and $\left\langle\hat{\varepsilon}_{\text {int }}(\vec{r})\right\rangle^{t}$ can be obtained in a standard way. Such a function has the following form [7, 9]:

$$
\varrho_{q}\left(x^{N} ; t\right)=\exp \left\{-\Phi(t)-\int \mathrm{d} \vec{r} \beta(\vec{r} ; t) \hat{\varepsilon}_{\text {int }}(\vec{r})-\int \mathrm{d} x a(x ; t) \hat{n}_{1}(x)\right\}
$$

where

$$
\Phi(t)=\ln \int \mathrm{d} \Gamma_{N} \exp \left\{-\int \mathrm{d} \vec{r} \beta(\vec{r} ; t) \hat{\varepsilon}_{\text {int }}(\vec{r})-\int \mathrm{d} x a(x ; t) \hat{n}_{1}(x)\right\}
$$


has been determined from the normalization condition for distribution function $\int \mathrm{d} \Gamma_{N} \varrho_{q}\left(x^{N} ; t\right)=$ 1. $\beta(\vec{r} ; t)$ and $a(x ; t)$ are the Lagrange multipliers, which are defined from the self-consistency conditions:

$$
\left\langle\hat{\varepsilon}_{\text {int }}(\vec{r})\right\rangle^{t}=\left\langle\hat{\varepsilon}_{\text {int }}(\vec{r})\right\rangle_{q}^{t}, \quad\left\langle\hat{n}_{1}(x)\right\rangle^{t}=\left\langle\hat{n}_{1}(x)\right\rangle_{q}^{t}
$$

In order to understand the physical sense of parameters $\beta(\vec{r} ; t)$ and $a(x ; t)$, let us transform the function in the following way

$$
\varrho_{q}\left(x^{N} ; t\right)=\exp \left\{-\Phi(t)-\int \mathrm{d} \vec{r} \beta(\vec{r} ; t) \hat{\varepsilon}^{\prime}(\vec{r})-\int \mathrm{d} x a^{\prime}(x ; t) \hat{n}_{1}(x)\right\},
$$

where

$$
\hat{\varepsilon}^{\prime}(\vec{r})=\hat{\varepsilon}_{\text {kin }}(\vec{r})-\vec{v}(\vec{r} ; t) \cdot \hat{\vec{\jmath}}(\vec{r})+\frac{m}{2} v^{2}(\vec{r} ; t) \hat{n}_{1}(\vec{r})+\hat{\varepsilon}_{\text {int }}(\vec{r})
$$

is the total energy density in the reference frame moving together with a system element of the mass velocity $\vec{v}(\vec{r} ; t)$. Herewith, the parameter $a^{\prime}(x ; t)$ is of the form

$$
a^{\prime}(x ; t)=a(x ; t)-\beta(\vec{r} ; t)\left\{\frac{p^{2}}{2 m}-\vec{v}(\vec{r} ; t) \cdot \vec{p}+\frac{m}{2} v^{2}(\vec{r} ; t)\right\} .
$$

Consequently, the first self-consistency condition (9) transforms to

$$
\left\langle\hat{\varepsilon}^{\prime}(\vec{r})\right\rangle^{t}=\left\langle\hat{\varepsilon}^{\prime}(\vec{r})\right\rangle_{q}^{t}
$$

When the self-consistency conditions (9) and (12) are fulfilled, we obtain the relations

$$
\frac{\delta \Phi(t)}{\delta \beta(\vec{r} ; t)}=-\left\langle\hat{\varepsilon}^{\prime}(\vec{r})\right\rangle^{t}, \quad \frac{\delta \Phi(t)}{\delta a^{\prime}(x ; t)}=-\left\langle\hat{n}_{1}(x)\right\rangle_{q}^{t}=-\left\langle\hat{n}_{1}(x)\right\rangle^{t}=-f_{1}(x ; t) .
$$

They mean that parameters $\beta(\vec{r} ; t)$ and $a^{\prime}(x ; t)$ are conjugated to $\left\langle\hat{\varepsilon}^{\prime}(\vec{r})\right\rangle^{t}$ and $\left\langle\hat{n}_{1}(x)\right\rangle^{t}$, respectively. Now let us determine the entropy of the system, taking into account the self-consistency conditions (9) and (12):

$$
S(t)=-\left\langle\ln \varrho_{q}\left(x^{N} ; t\right)\right\rangle_{q}^{t}=\Phi(t)+\int \mathrm{d} \vec{r} \beta(\vec{r} ; t)\left\langle\hat{\varepsilon}^{\prime}(\vec{r})\right\rangle^{t}+\int \mathrm{d} x a^{\prime}(x ; t)\left\langle\hat{n}_{1}(x)\right\rangle^{t} .
$$

Then, the thermodynamic relations can be obtained as

$$
\frac{\delta S(t)}{\delta\left\langle\hat{\varepsilon}^{\prime}(\vec{r})\right\rangle^{t}}=\beta(\vec{r} ; t), \quad \frac{\delta S(t)}{\delta\left\langle\hat{n}_{1}(x)\right\rangle^{t}}=a^{\prime}(x ; t) .
$$

From relations (15) we see that parameter $\beta(\vec{r} ; t)$ can be considered as an analogue of the local inverse temperature. Excluding $a^{\prime}(x ; t)$ by means of self-consistency condition (9) in (10), one obtains an explicit expression for the entropy of a nonequilibrium state when kinetics and hydrodynamics are considered consistently [3, 7]:

$$
S(t)=\Phi(t)+\int \mathrm{d} \vec{r} \beta(\vec{r} ; t)\left\{\langle\hat{\varepsilon}(\vec{r})\rangle^{t}-\vec{v}(\vec{r} ; t)\langle\hat{\vec{\jmath}}(\vec{r})\rangle^{t}+\frac{m}{2} v^{2}(\vec{r} ; t)\langle\hat{n}(\vec{r})\rangle^{t}\right\}-\int \mathrm{d} x f_{1}(x ; t) \ln \frac{f_{1}(x ; t)}{u(\vec{r} ; t)},(
$$

and

$$
u(\vec{r} ; t)=\int \frac{\mathrm{d} \vec{r}^{N-1}}{(N-1) !} \exp \left\{-\Phi(t)-\int \mathrm{d} \vec{r} \beta(\vec{r} ; t) \hat{\varepsilon}^{\prime}(\vec{r})\right\} \prod_{j=2}^{N} \frac{n\left(\vec{r}_{j} ; t\right)}{u\left(\vec{r}_{j} ; t\right)}
$$

Explicit contributions of the averaged values of hydrodynamic variables $\langle\hat{\varepsilon}(\vec{r})\rangle^{t},\langle\hat{\vec{\jmath}}(\vec{r})\rangle^{t}$, and $\langle\hat{n}(\vec{r})\rangle^{t}$ and the contribution of kinetics in the form analogous to the Boltzmann entropy (the last term) 
are present in the expression (16). However, if we formally omit the contribution of potential energy, it is possible to show that $u(\vec{r} ; t)=\mathrm{e}$ and the expression (16) explicitly transforms into the Boltzmann entropy. For the quasiequilibrium statistical operator (10) the Kawasaki-Gunton projection operator $P_{q}(t)$ has the following form:

$$
\begin{aligned}
P_{q}(t) \varrho^{\prime}\left(x^{N} ; t\right)= & \left\{\varrho_{q}\left(x^{N} ; t\right)-\int \mathrm{d} \vec{r} \frac{\delta \varrho_{q}\left(x^{N} ; t\right)}{\delta\left\langle\hat{\varepsilon}^{\prime}(\vec{r})\right\rangle^{t}}\left\langle\hat{\varepsilon}^{\prime}(\vec{r})\right\rangle^{t}-\int \mathrm{d} x \frac{\delta \varrho_{q}\left(x^{N} ; t\right)}{\delta\left\langle\hat{n}_{1}(x)\right\rangle^{t}}\left\langle\hat{n}_{1}(x)\right\rangle^{t}\right\} \int \mathrm{d} \Gamma_{N} \varrho^{\prime}\left(x^{N} ; t\right) \\
& +\int \mathrm{d} \vec{r} \frac{\delta \varrho_{q}\left(x^{N} ; t\right)}{\delta\left\langle\hat{\varepsilon}^{\prime}(\vec{r})\right\rangle^{t}} \int \mathrm{d} \Gamma_{N} \hat{\varepsilon}^{\prime}(\vec{r}) \varrho^{\prime}\left(x^{N} ; t\right)+\int \mathrm{d} \vec{r} \frac{\delta \varrho_{q}\left(x^{N} ; t\right)}{\delta\left\langle\hat{n}_{1}(x)\right\rangle^{t}} \int \mathrm{d} \Gamma_{N} \hat{n}_{1}(x) \varrho^{\prime}\left(x^{N} ; t\right) .
\end{aligned}
$$

The projection operator $P_{q}(t)$ acts on distribution functions only and possesses the following properties: $P_{q}(t) P_{q}\left(t^{\prime}\right)=P_{q}(t), \quad P_{q}(t) \varrho_{q}\left(t^{\prime}\right)=\varrho_{q}\left(t^{\prime}\right), \quad P_{q}(t) \varrho\left(t^{\prime}\right)=\varrho_{q}\left(t^{\prime}\right)$.

The solution (6) is exact and corresponds to the idea of a reduced description of kinetic and hydrodynamic processes of nonequilibrium states of the system. In [9] by means of $\varrho\left(x^{N} ; t\right)(\underline{6})$ a set of the coupled equations for a nonequilibrium one-particle distribution function and the averaged density of the total energy was obtained. This set is strongly nonlinear. It takes into account complicated kinetic and hydrodynamic processes and can be used for the description of both strongly and weakly nonequilibrium states. Now we will show that in the case of nonequilibrium sates which are close to equilibrium these equations can be significantly simplified.

\section{Equations for a consistent description of kinetics and hydrodynamics in weakly nonequilibrium states}

Studying the nonequilibrium states of that system which is close enough to equilibrium it is quite natural to suppose that the averaged value of energy $\langle\hat{\varepsilon}(\vec{r})\rangle^{t}$, the nonequilibrium distribution function $f_{1}(x ; t)$ as well as the parameters $\beta(\vec{r} ; t)$ and $b(x ; t)=a(x ; t)-\beta(\vec{r} ; t) p^{2} / 2 m$ slightly differ from their corresponding equilibrium values. Then, we can expand the quasiequilibrium distribution function (10)

$$
\varrho_{q}\left(x^{N} ; t\right)=\exp \left\{-\Phi(t)-\int \mathrm{d} \vec{r} \beta(\vec{r} ; t) \hat{\varepsilon}(\vec{r})-\int \mathrm{d} x b(x ; t) \hat{n}_{1}(x)\right\}
$$

into a series with respect to deviations of parameters $\delta \beta(\vec{r} ; t)=\beta(\vec{r} ; t)-\beta$ and $\delta b(x ; t)=b(x ; t)-\beta \mu$, and restrict the consideration to the linear approximation:

$$
\varrho_{q}\left(x^{N} ; t\right)=\varrho_{0}\left(x^{N}\right)\left\{1-\int \mathrm{d} \vec{r} \delta \beta(\vec{r} ; t) \hat{\varepsilon}(\vec{r})-\int \mathrm{d} x \delta b(x ; t) \hat{n}_{1}(x)\right\} .
$$

Here, $\beta=1 / k_{\mathrm{B}} T, T$ is the equilibrium value of temperature, $\varrho_{0}\left(x^{N}\right)=Z^{-1} \mathrm{e}^{-\beta(H-\mu N)}$ is the equilibrium distribution function, $Z=\int \mathrm{d} \Gamma_{N} \mathrm{e}^{-\beta(H-\mu N)}$ is the grand partition function, $\mu$ is the equilibrium value of chemical potential. The values of parameters $\delta b(x ; t)$ and $\delta \beta(\vec{r} ; t)$ are determined from the self-consistency conditions:

$$
\langle\hat{\varepsilon}(\vec{r})\rangle^{t}=\langle\hat{\varepsilon}(\vec{r})\rangle_{q}^{t}, \quad\left\langle\hat{n}_{1}(x)\right\rangle^{t}=\left\langle\hat{n}_{1}(x)\right\rangle_{q}^{t} .
$$

Then, using the Fourier transformation for spatial coordinates and self-consistency equations (19), we write down the distribution function (18) in the form [9]:

$\varrho_{q}\left(x^{N} ; t\right)=\varrho_{0}\left(x^{N}\right)\left\{1+\sum_{\vec{k}}^{\prime}\left\langle\hat{h}_{\vec{k}}^{\mathrm{int}}\right\rangle^{t} \Phi_{h h}^{-1}(\vec{k}) \hat{h}_{\vec{k}}^{\mathrm{int}}+\sum_{\vec{k}}^{\prime} \int \mathrm{d} \vec{p} \int \mathrm{d} \vec{p}^{\prime}\left\langle\hat{n}_{\vec{k}}(\vec{p})\right\rangle^{t} \Phi_{\vec{k}}^{-1}\left(\vec{p}, \vec{p}^{\prime}\right) \hat{n}_{\vec{k}}\left(\vec{p}^{\prime}\right)\right\}$,

where $\sum_{\vec{k}}{ }^{\prime}=\sum_{\vec{k}(\vec{k} \neq 0)}, \vec{k}$ is the wave-vector.

$$
\hat{n}_{\vec{k}}(\vec{p})=\int \mathrm{d} \vec{r} \mathrm{e}^{-\mathrm{i} \vec{k} \vec{r}} \hat{n}_{1}(\vec{r}, \vec{p})
$$


is the Fourier-components of microscopic phase density of particles number,

$$
\hat{h}_{\vec{k}}^{\text {int }}=\hat{\varepsilon}_{\vec{k}}^{\text {int }}-\left\langle\hat{\varepsilon}_{\vec{k}}^{\text {int }} \hat{n}_{-\vec{k}}\right\rangle_{0} S_{2}^{-1}(k) \hat{n}_{\vec{k}}
$$

the Fourier-components of the potential part of the enthalpy density, $\hat{\varepsilon}_{\vec{k}}^{\mathrm{int}}=\frac{1}{2} \sum_{l \neq j=1}^{N} \Phi\left(\left|\vec{r}_{l j}\right|\right) \mathrm{e}^{-\mathrm{i} \vec{k} \vec{r}_{j}}$ and $\hat{n}_{\vec{k}}=\sum_{l=1}^{N} \mathrm{e}^{-\mathrm{i} \vec{k} \vec{r}_{l}}$ are the Fourier-components of the potential energy and particle number densities, respectively. $\Phi_{h h}^{-1}(\vec{k})$ is the function inverse to the equilibrium correlation function $\Phi_{h h}(\vec{k})=\left\langle\hat{h}_{\vec{k}}^{\mathrm{int}} \hat{h}_{-\vec{k}}^{\mathrm{int}}\right\rangle_{0},\langle\ldots\rangle_{0}=\int \mathrm{d} \Gamma_{N} \ldots \varrho_{0}\left(x^{N}\right),\left\langle\hat{n}_{\vec{k}}(\vec{p})\right\rangle_{0}=0(\vec{k} \neq 0) . \Phi_{\vec{k}}^{-1}\left(\vec{p}, \vec{p}^{\prime}\right)$ is the function inverse to $\Phi_{\vec{k}}\left(\vec{p}, \vec{p}^{\prime}\right)=\left\langle\hat{n}_{\vec{k}}(\vec{p}) \hat{n}_{-\vec{k}}\left(\vec{p}^{\prime}\right)\right\rangle_{0}=n \delta\left(\vec{p}-\vec{p}^{\prime}\right) f_{0}\left(p^{\prime}\right)+n^{2} f_{0}(p) f_{0}\left(p^{\prime}\right) h_{2}(\vec{k})$. It is equal to

$$
\Phi_{\vec{k}}^{-1}\left(\vec{p}, \vec{p}^{\prime}\right)=\frac{\delta\left(\vec{p}-\vec{p}^{\prime}\right)}{n f_{0}\left(p^{\prime}\right)}-c_{2}(k),
$$

where $n=N / V, f_{0}(p)=(\beta / 2 \pi m)^{3 / 2} \exp \left(-\beta p^{2} / 2 m\right)$ is the Maxwellian distribution and $c_{2}(k)$ is the direct correlation function, related to correlation function $h_{2}(k): h_{2}(k)=c_{2}(k)\left[1-n c_{2}(k)\right]^{-1}$. $S_{2}(k)=\left\langle\hat{n}_{\vec{k}} \hat{n}_{-\vec{k}}\right\rangle_{0}$ is the static structure factor. It is important to note that dynamic variables $\hat{h}_{\vec{k}}^{\text {int }}$ and $\hat{n}_{\vec{k}}(\vec{p})$ in the distribution (20) are orthogonal in the sense that $\left\langle\hat{h}_{\vec{k}}^{\text {int }} \hat{n}_{\vec{k}}(\vec{p})\right\rangle_{0}=0$. In the approximation (20), the nonequilibrium distribution function $\varrho\left(x^{N} ; t\right)$ (6) has the following form:

$$
\begin{aligned}
\varrho\left(x^{N} ; t\right)= & \varrho_{0}\left(x^{N}\right)\left\{1+\sum_{\vec{k}}^{\prime} \int \mathrm{d} \vec{p} \int \mathrm{d} \vec{p}^{\prime}\left\langle\hat{n}_{\vec{k}}(\vec{p})\right\rangle^{t} \Phi_{\vec{k}}^{-1}\left(\vec{p}, \vec{p}^{\prime}\right) \hat{n}_{-\vec{k}}\left(\vec{p}^{\prime}\right)+\sum_{\vec{k}}^{\prime}\left\langle\hat{h}_{\vec{k}}^{\text {int }}\right\rangle^{t} \Phi_{h h}^{-1}(\vec{k}) \hat{h}_{-\vec{k}}^{\text {int }}\right. \\
& -\sum_{\vec{k}}^{\prime} \int \mathrm{d} \vec{p} \int \mathrm{d} \vec{p}^{\prime} \int_{-\infty}^{t} \mathrm{e}^{\varepsilon\left(t^{\prime}-t\right)} T_{0}\left(t, t^{\prime}\right) I_{n}(-\vec{k} ; \vec{p}) \Phi_{\vec{k}}^{-1}\left(\vec{p}, \vec{p}^{\prime}\right)\left\langle\hat{n}_{\vec{k}}\left(\vec{p}^{\prime}\right)\right\rangle^{t^{\prime}} \mathrm{d} t^{\prime} \\
& \left.-\sum_{\vec{k}}^{\prime} \int_{-\infty}^{t} \mathrm{e}^{\varepsilon\left(t^{\prime}-t\right)} T_{0}\left(t, t^{\prime}\right) I_{h}^{\text {int }}(-\vec{k}) \Phi_{h h}^{-1}(\vec{k})\left\langle\hat{h}_{\vec{k}}^{\text {int }}\right\rangle^{t^{\prime}} \mathrm{d} t^{\prime}\right\},
\end{aligned}
$$

where

$$
I_{n}(\vec{k} ; \vec{p})=\left(1-P_{0}\right) \mathrm{i} L_{N} \hat{n}_{\vec{k}}(\vec{p})=\left(1-P_{0}\right) \dot{\hat{n}}_{\vec{k}}(\vec{p}), \quad I_{h}^{\text {int }}(\vec{k})=\left(1-P_{0}\right) \text { i } L_{N} \hat{h}_{\vec{k}}^{\text {int }}=\left(1-P_{0}\right) \dot{\hat{h}}_{\vec{k}}^{\text {int }}
$$

are the generalized flows in linear approximation and $T_{0}\left(t, t^{\prime}\right)=\exp \left[\left(t-t^{\prime}\right)\left(1-P_{0}\right) \mathrm{i} L_{N}\right]$ the evolution operator with regard to projection $P_{0}$. Operator $P_{0}$ is the linear approximation of the Mori projection operator and is constructed on the orthogonal dynamic variables $\hat{n}_{\vec{k}}(\vec{p}), \hat{h}_{\vec{k}}^{\text {int }}[9]$ :

$$
P_{0} \hat{A}_{\vec{k}}=\sum_{\vec{k}}^{\prime}\left\langle\hat{A}_{\vec{k}} \hat{h}_{-\vec{k}}^{\mathrm{int}}\right\rangle_{0} \Phi_{h h}^{-1}(\vec{k}) \hat{h}_{\vec{k}}^{\mathrm{int}}+\sum_{\vec{k}}^{\prime} \int \mathrm{d} \vec{p} \int \mathrm{d} \vec{p}^{\prime}\left\langle\hat{A}_{\vec{k}} \hat{n}_{-\vec{k}}(\vec{p})\right\rangle_{0} \Phi_{\vec{k}}^{-1}\left(\vec{p}, \vec{p}^{\prime}\right) \hat{n}_{\vec{k}}\left(\vec{p}^{\prime}\right)
$$

It possesses the properties $P_{0} P_{0}=P_{0}, P_{0}\left(1-P_{0}\right)=0, P_{0} \hat{n}_{\vec{k}}(\vec{p})=\hat{n}_{\vec{k}}(\vec{p}), P_{0} \hat{h}_{\vec{k}}^{\text {int }}=\hat{h}_{\vec{k}}^{\text {int }}$.

In view of its own structure, the nonequilibrium distribution function (24) is a functional of the reduced-description parameters $\left\langle\hat{n}_{\vec{k}}(\vec{p})\right\rangle^{t},\left\langle\hat{h}_{\vec{k}}^{\text {int }}\right\rangle^{t}$, dynamic variables $\hat{n}_{\vec{k}}(\vec{p}), \hat{h}_{\vec{k}}^{\text {int }}$ along with their generalized flows (25). Using $\varrho\left(x^{N} ; t\right)$ (24) for the parameters of reduced description $f_{\vec{k}}(\vec{p} ; t)=$ $\left\langle\hat{n}_{\vec{k}}(\vec{p})\right\rangle^{t}, h_{\vec{k}}^{\text {int }}(t)=\left\langle\hat{h}_{\vec{k}}^{\text {int }}\right\rangle^{t}$ we can obtain the following set of equations $[9]$ :

$$
\begin{aligned}
& \frac{\partial}{\partial t} f_{\vec{k}}(\vec{p} ; t)+\frac{\mathrm{i} \vec{k} \cdot \vec{p}}{m} f_{\vec{k}}(\vec{p} ; t)=-\frac{\mathrm{i} \vec{k} \cdot \vec{p}}{m} n f_{0}(p) c_{2}(k) \int \mathrm{d} \vec{p}^{\prime} f_{\vec{k}}\left(\vec{p}^{\prime} ; t\right)+\mathrm{i} \Omega_{n h}(\vec{k} ; \vec{p}) h_{\vec{k}}^{\mathrm{int}}(t) \\
& \quad-\int \mathrm{d} \vec{p}^{\prime} \int_{-\infty}^{t} \mathrm{e}^{\varepsilon\left(t^{\prime}-t\right)} \varphi_{n n}\left(\vec{k} ; \vec{p}, \vec{p}^{\prime} ; t, t^{\prime}\right) f_{\vec{k}}\left(\vec{p}^{\prime} ; t^{\prime}\right) \mathrm{d} t^{\prime}-\int_{-\infty}^{t} \mathrm{e}^{\varepsilon\left(t^{\prime}-t\right)} \varphi_{n h}\left(\vec{k} ; \vec{p} ; t, t^{\prime}\right) h_{\vec{k}}^{\mathrm{int}}\left(t^{\prime}\right) \mathrm{d} t^{\prime},
\end{aligned}
$$




$$
\begin{aligned}
\frac{\partial}{\partial t} h_{\vec{k}}^{\text {int }}(t)= & \int \mathrm{d} \vec{p}^{\prime} \mathrm{i} \Omega_{h n}\left(\vec{k} ; \vec{p}^{\prime}\right) f_{\vec{k}}\left(\vec{p}^{\prime} ; t\right)-\int \mathrm{d} \vec{p}^{\prime} \int_{-\infty}^{t} \mathrm{e}^{\varepsilon\left(t^{\prime}-t\right)} \varphi_{h n}\left(\vec{k} ; \vec{p}^{\prime} ; t, t^{\prime}\right) f_{\vec{k}}\left(\vec{p}^{\prime} ; t^{\prime}\right) \mathrm{d} t^{\prime} \\
& -\int_{-\infty}^{t} \mathrm{e}^{\varepsilon\left(t^{\prime}-t\right)} \varphi_{h h}\left(\vec{k} ; t, t^{\prime}\right) h_{\vec{k}}^{\text {int }}\left(t^{\prime}\right) \mathrm{d} t^{\prime} .
\end{aligned}
$$

Here, $\mathrm{i} \Omega_{n h}(\vec{k} ; \vec{p}), \mathrm{i} \Omega_{h n}(\vec{k} ; \vec{p})$ are the normalized static correlation functions

$$
\mathrm{i} \Omega_{n h}(\vec{k} ; \vec{p})=\left\langle\dot{\hat{n}}_{\vec{k}}(\vec{p}) \hat{h}_{-\vec{k}}^{\mathrm{int}}\right\rangle_{0} \Phi_{h h}^{-1}(\vec{k}), \quad \mathrm{i} \Omega_{h n}(\vec{k} ; \vec{p})=\int \mathrm{d} \vec{p}^{\prime}\left\langle\dot{\hat{h}}_{\vec{k}}^{\mathrm{int}} \hat{n}_{-\vec{k}}\left(\vec{p}^{\prime}\right)\right\rangle_{0} \Phi_{\vec{k}}^{-1}\left(\vec{p}^{\prime}, \vec{p}\right)
$$

and

$$
\begin{gathered}
\varphi_{n n}\left(\vec{k} ; \vec{p}, \vec{p}^{\prime} ; t, t^{\prime}\right)=\int \mathrm{d} \vec{p}^{\prime \prime}\left\langle I_{n}(\vec{k} ; \vec{p}) T_{0}\left(t, t^{\prime}\right) I_{n}\left(-\vec{k} ; \vec{p}^{\prime \prime}\right)\right\rangle_{0} \Phi_{\vec{k}}^{-1}\left(\vec{p}^{\prime \prime}, \vec{p}^{\prime}\right), \\
\varphi_{h n}\left(\vec{k} ; \vec{p} ; t, t^{\prime}\right)=\int \mathrm{d} \vec{p}^{\prime}\left\langle I_{h}^{\operatorname{int}}(\vec{k}) T_{0}\left(t, t^{\prime}\right) I_{n}\left(-\vec{k} ; \vec{p}^{\prime}\right)\right\rangle_{0} \Phi_{\vec{k}}^{-1}\left(\vec{p}^{\prime}, \vec{p}\right), \\
\varphi_{n h}\left(\vec{k} ; \vec{p} ; t, t^{\prime}\right)=\left\langle I_{n}(\vec{k} ; \vec{p}) T_{0}\left(t, t^{\prime}\right) I_{h}^{\text {int }}(-\vec{k})\right\rangle_{0} \Phi_{h h}^{-1}(\vec{k}) \\
\varphi_{h h}\left(\vec{k} ; \vec{p} ; t, t^{\prime}\right)=\left\langle I_{h}^{\operatorname{int}}(\vec{k}) T_{0}\left(t, t^{\prime}\right) I_{h}^{\text {int }}(-\vec{k})\right\rangle_{0} \Phi_{h h}^{-1}(\vec{k})
\end{gathered}
$$

are the generalized transport kernels (memory functions) describing kinetic and hydrodynamic processes. The set of equations (27) and (28) is closed with respect to the parameters of reduced description $f_{\vec{k}}(\vec{p} ; t), h_{\vec{k}}^{\text {int }}(t)$. If in this set of equations one formally puts $\hat{h}_{\vec{k}}^{\text {int }}=0$, then we obtain the kinetic equation for $f_{\vec{k}}(\vec{p} ; t)$ :

$$
\begin{aligned}
\frac{\partial}{\partial t} f_{\vec{k}}(\vec{p} ; t)+\frac{\mathrm{i} \vec{k} \cdot \vec{p}}{m} f_{\vec{k}}(\vec{p} ; t)= & -\frac{\mathrm{i} \vec{k} \cdot \vec{p}}{m} n f_{0}(p) c_{2}(k) \int \mathrm{d} \vec{p}^{\prime} f_{\vec{k}}\left(\vec{p}^{\prime} ; t\right) \\
& -\int \mathrm{d} \vec{p}^{\prime} \int_{-\infty}^{t} \mathrm{e}^{\varepsilon\left(t^{\prime}-t\right)} \varphi_{n n}^{\prime}\left(\vec{k} ; \vec{p}, \vec{p}^{\prime} ; t, t^{\prime}\right) f_{\vec{k}}\left(\vec{p}^{\prime} ; t^{\prime}\right) \mathrm{d} t^{\prime} .
\end{aligned}
$$

This is true when the contribution from the potential energy is considerably smaller than the averaged kinetic energy (e.g. in the case of gases or weakly coupled liquids). The equation (31) was obtained for the first time by means of the Mori projection operators method in [13 15]. Therein, the basic parameter of the reduced description was a nonequilibrium one-particle distribution function $f_{\vec{k}}(\vec{p} ; t)$ which corresponds to the microscopic phase density $\hat{n}_{\vec{k}}(\vec{p})$ (the Klimontovich function). In this case the memory function $\varphi_{n n}^{\prime}\left(\vec{k} ; \vec{p}, \vec{p}^{\prime} ; t, t^{\prime}\right)$ has the following structure:

$$
\varphi_{n n}^{\prime}\left(\vec{k} ; \vec{p}, \vec{p}^{\prime} ; t, t^{\prime}\right)=\int \mathrm{d} \vec{p}^{\prime \prime}\left\langle I_{n}^{0}(\vec{k}, \vec{p}) T_{0}^{\prime}\left(t, t^{\prime}\right) I_{n}^{0}\left(-\vec{k}, \vec{p}^{\prime \prime}\right)\right\rangle_{0} \Phi_{\vec{k}}^{-1}\left(\vec{p}^{\prime \prime}, \vec{p}^{\prime}\right),
$$

where $I_{n}^{0}(\vec{k}, \vec{p})=\left(1-P_{0}^{\prime}\right) \dot{\hat{n}}_{\vec{k}}(\vec{p})$ is the generalized flow, $P_{0}^{\prime}$ is the Mori operator, introduced in 13 15]

$$
P_{0}^{\prime} \hat{A}_{\vec{k}^{\prime}}=\sum_{\vec{k}}^{\prime} \int \mathrm{d} \vec{p} \int \mathrm{d} \vec{p}^{\prime}\left\langle\hat{A}_{\vec{k}^{\prime}} \hat{n}_{-\vec{k}^{\prime}}\left(\vec{p}^{\prime}\right)\right\rangle_{0} \Phi_{\vec{k}}^{-1}\left(\vec{p}^{\prime}, \vec{p}\right) \hat{n}_{\vec{k}}(\vec{p})
$$

and $T_{0}^{\prime}\left(t, t^{\prime}\right)$ is the corresponding evolution operator with regard to projection. Based on the kinetic equation (31), the investigations of the dynamic structure factor, transverse and longitudinal current time correlation functions, diffusion and viscosity coefficients for dense gases and liquids where carried out [1, 2, 13 24, 26]. In particular, in Mazenko's papers the linearized BoltzmannEnskog equation was obtained by means of expansion of the memory functions $\varphi_{n n}^{\prime}\left(\vec{k} ; \vec{p}, \vec{p}^{\prime} ; t, t^{\prime}\right)$ in density. For the case of weak coupling, the equation of Fokker-Planck type was obtained. However, the main drawback of kinetic equation (31) consists in its inconsistency with the total energy conservation law, especially for dense gases and liquids, when the contribution of potential energy 
into thermodynamic functions and transport coefficients is determinant. In [1] this drawback was studied in detail. Therein, the investigations of the dynamic structure factor at intermediate values of wave-vector $\vec{k}$ and frequency $\omega$ for simple liquids were carried out by using the Mori projection operators method for the reduced-description parameters $\hat{n}_{\vec{k}}(\vec{p}), \hat{\varepsilon}_{\vec{k}}$. Contrary to the transport equations presented in [1], our set of equations (27) and (28) is constructed on the orthogonal dynamic variables $\hat{n}_{\vec{k}}(\vec{p}), \hat{h}_{\vec{k}}^{\text {int }}$. Therefore, "kinetic" and "hydrodynamic" contributions are separated and correlation between them is described by the generalized memory functions (30). It is important to reveal their inner structure.

\section{Memory functions of a consistent description of kinetic and hydrody- namic processes}

In order to study the structure of the memory functions (30) let us look at the form of the corresponding generalized flows (25) on which the memory functions are built. In particular, let us take into consideration the fact that

$$
\dot{\hat{n}}_{\vec{k}}(\vec{p})=\mathrm{i} L_{N} \hat{n}_{\vec{k}}(\vec{p})=-\frac{\mathrm{i} \vec{k}}{m} \cdot \hat{\vec{\jmath}}_{\vec{k}}(\vec{p})+\frac{\partial}{\partial \vec{p}} \cdot \vec{F}_{\vec{k}}(\vec{p}),
$$

where

$$
\hat{\vec{J}}_{\vec{k}}(\vec{p})=\sum_{j=1}^{N} \vec{p}_{j} \mathrm{e}^{-\mathrm{i} \vec{k} \cdot \vec{r}_{j}} \delta\left(\vec{p}-\vec{p}_{j}\right)
$$

is the momentum density in the $(\vec{k}, \vec{p})$ space and

$$
\vec{F}_{\vec{k}}(\vec{p})=\frac{1}{2} \sum_{j \neq l} \frac{\partial}{\partial \vec{r}_{j}} \cdot \Phi_{\left|\vec{r}_{j}-\vec{r}_{l}\right|} \delta\left(\vec{p}-\vec{p}_{j}\right) \mathrm{e}^{-\mathrm{i} \vec{k}_{r_{j}}}
$$

The action of the Mori projection operator $P_{0}$ on $\dot{\hat{n}}_{\vec{k}}(\vec{p})$ can be presented as

$$
P_{0} \dot{\hat{n}}_{\vec{k}}(\vec{p})=-\frac{\beta}{m} \vec{p} \cdot \vec{\Phi}_{F h}(\vec{k}) \hat{h}_{\vec{k}}^{\mathrm{int}} f_{0}(p)-\frac{\mathrm{i} \vec{k}}{m} \cdot \vec{p} \hat{n}_{\vec{k}}(\vec{p})
$$

where

$$
\vec{\Phi}_{F h}(\vec{k})=\left\langle\vec{F}_{\vec{k}} \hat{h}_{\vec{k}}^{\text {int }}\right\rangle_{0} \Phi_{h h}^{-1}(\vec{k})
$$

Taking into account (23) we write down the memory function $\varphi_{n n}\left(\vec{k} ; \vec{p}, \vec{p}^{\prime} ; t, t^{\prime}\right)$ in the following form

$$
\begin{aligned}
& \varphi_{n n}\left(\vec{k} ; \vec{p}, \vec{p}^{\prime} ; t, t^{\prime}\right)=\int \mathrm{d} \vec{p}^{\prime \prime}\left\langle I_{n}(\vec{k} ; \vec{p}) T_{0}\left(t, t^{\prime}\right) I_{n}\left(-\vec{k} ; \vec{p}^{\prime \prime}\right)\right\rangle_{0}\left\{\frac{\delta\left(\vec{p}^{\prime \prime}-\vec{p}^{\prime}\right)}{n f_{0}\left(p^{\prime}\right)}-c_{2}(k)\right\} \\
& \quad=\left\langle I_{n}(\vec{k} ; \vec{p}) T_{0}\left(t, t^{\prime}\right) I_{n}\left(-\vec{k} ; \vec{p}^{\prime}\right)\right\rangle_{0} \frac{1}{n f_{0}\left(p^{\prime}\right)}-\int \mathrm{d} \vec{p}^{\prime \prime}\left\langle I_{n}(\vec{k} ; \vec{p}) T_{0}\left(t, t^{\prime}\right) I_{n}\left(-\vec{k} ; \vec{p}^{\prime \prime}\right)\right\rangle_{0} c_{2}(k) .
\end{aligned}
$$

The second term in the right-hand side of (39) is equal to zero, because

$$
\int \mathrm{d} \vec{p} I_{n}(\vec{k} ; \vec{p})=0
$$

The transport kernel $\varphi_{n n}\left(\vec{k} ; \vec{p}, \vec{p}^{\prime} ; t, t^{\prime}\right)$ enters the kinetic equation (27) as the term $\int \varphi_{n n}\left(\vec{k} ; \vec{p}, \vec{p}^{\prime} ; t, t^{\prime}\right) f_{\vec{k}}\left(\vec{p}^{\prime} ; t\right) \mathrm{d} \vec{p}^{\prime}$. Taking into account (39) and (40) we can write the last one in 
the form

$$
\begin{aligned}
\int \mathrm{d} \vec{p}^{\prime} \varphi_{n n}\left(\vec{k} ; \vec{p}, \vec{p}^{\prime} ; t, t^{\prime}\right) f_{\vec{k}}\left(\vec{p}^{\prime} ; t\right) & =\int \mathrm{d} \vec{p}^{\prime}\left\{\bar{\varphi}_{\jmath \jmath}\left(\vec{k} ; \vec{p}, \vec{p}^{\prime} ; t, t^{\prime}\right)-\frac{\partial}{\partial \vec{p}} \varphi_{F F}\left(\vec{k} ; \vec{p}, \vec{p}^{\prime} ; t, t^{\prime}\right)\right. \\
& \left.\times\left(\frac{\beta}{m n f_{0}\left(p^{\prime}\right)} \vec{p}^{\prime}-\frac{\partial}{\partial \vec{p}^{\prime}}\right)\right\} f_{\vec{k}}\left(\vec{p}^{\prime} ; t^{\prime}\right)-\bar{\varphi}_{n \jmath}^{(2)}\left(\vec{k} ; \vec{p} ; t, t^{\prime}\right) \cdot\left\langle\hat{\vec{\jmath}}_{\vec{k}}\right\rangle^{t},
\end{aligned}
$$

where the second term has the structure of a generalized Fokker-Planck operator containing the generalized friction coefficient $\varphi_{F F}\left(\vec{k} ; \vec{p}, \vec{p}^{\prime} ; t, t^{\prime}\right)$ in the spatially-impulse space.

$$
\begin{aligned}
\bar{\varphi}_{\jmath \jmath}\left(\vec{k} ; \vec{p}, \vec{p}^{\prime} ; t, t^{\prime}\right)= & \frac{\vec{k}}{m} \cdot \varphi_{\jmath \jmath}\left(\vec{k} ; \vec{p}, \vec{p}^{\prime} ; t, t^{\prime}\right) \cdot \frac{\vec{k}}{m}-\frac{\mathrm{i} \vec{k}}{m} \cdot \varphi_{\jmath F}\left(\vec{k} ; \vec{p}, \vec{p}^{\prime} ; t, t^{\prime}\right) \cdot \frac{\partial}{\partial \vec{p}^{\prime}} \\
& +\frac{\partial}{\partial \vec{p}} \cdot \varphi_{F \jmath}\left(\vec{k} ; \vec{p}, \vec{p}^{\prime} ; t, t^{\prime}\right) \cdot \frac{\mathrm{i} \vec{k}}{m}+\bar{\varphi}_{n n}^{(1)}\left(\vec{k} ; \vec{p}, \vec{p}^{\prime} ; t, t^{\prime}\right),
\end{aligned}
$$

$\varphi_{F F}\left(\vec{k} ; \vec{p}, \vec{p}^{\prime} ; t, t^{\prime}\right)=\left\langle\vec{F}_{\vec{k}}(\vec{p}) T_{0}\left(t, t^{\prime}\right) \vec{F}_{-\vec{k}}\left(\vec{p}^{\prime}\right)\right\rangle_{0}, \quad \varphi_{\jmath j}\left(\vec{k} ; \vec{p}, \vec{p}^{\prime} ; t, t^{\prime}\right)=\left\langle\hat{\vec{\jmath}_{\vec{k}}}(\vec{p}) T_{0}\left(t, t^{\prime}\right) \hat{\vec{\jmath}}_{-\vec{k}}\left(\vec{p}^{\prime}\right)\right\rangle_{0}$,

$\varphi_{\jmath F}\left(\vec{k} ; \vec{p}, \vec{p}^{\prime} ; t, t^{\prime}\right)=\left\langle\hat{\vec{\jmath}}_{\vec{k}}(\vec{p}) T_{0}\left(t, t^{\prime}\right) \vec{F}_{-\vec{k}}\left(\vec{p}^{\prime}\right)\right\rangle_{0}, \quad \varphi_{F \jmath}\left(\vec{k} ; \vec{p}, \vec{p}^{\prime} ; t, t^{\prime}\right)=\left\langle\vec{F}_{\vec{k}}(\vec{p}) T_{0}\left(t, t^{\prime}\right) \hat{\vec{\jmath}}_{-\vec{k}}\left(\vec{p}^{\prime}\right)\right\rangle_{0}$,

moreover,

$$
\int \mathrm{d} \vec{p} \int \mathrm{d} \vec{p}^{\prime} \varphi_{\jmath \jmath}\left(\vec{k} ; \vec{p}, \vec{p}^{\prime} ; t, t^{\prime}\right)=D\left(\vec{k} ; t, t^{\prime}\right)
$$

is the generalized coefficient of diffusion of particles and $\varphi_{\jmath \jmath}\left(\vec{k} ; \vec{p}, \vec{p}^{\prime} ; t, t^{\prime}\right)$ is the generalized diffusion coefficient in momentum space.

$$
\int \mathrm{d} \vec{p} \int \mathrm{d} \vec{p}^{\prime} \varphi_{F F}\left(\vec{k} ; \vec{p}, \vec{p}^{\prime} ; t, t^{\prime}\right)=\xi\left(\vec{k} ; t, t^{\prime}\right)
$$

is the generalized friction coefficient and $\varphi_{F F}\left(\vec{k} ; \vec{p}, \vec{p}^{\prime} ; t, t^{\prime}\right)$ is the generalized friction coefficient in momentum space.

$$
\begin{aligned}
\bar{\varphi}_{n n}^{(1)}\left(\vec{k} ; \vec{p}, \vec{p}^{\prime} ; t, t^{\prime}\right)=\frac{\beta}{m} \vec{p} \cdot \vec{\Phi}_{F h}(\vec{k}) f_{0}(p) \varphi_{h J}\left(\vec{k} ; \vec{p}^{\prime} ; t, t^{\prime}\right) \cdot \frac{\mathrm{i} \vec{k}}{m} \\
\quad-\frac{\vec{k}}{m} \cdot \vec{p} \varphi_{n J}\left(\vec{k} ; \vec{p}, \vec{p}^{\prime} ; t, t^{\prime}\right) \cdot \frac{\vec{k}}{m} \frac{\beta}{m} \vec{p} \cdot \vec{\Phi}_{F h}(\vec{k}) f_{0}(p) \varphi_{h F}\left(\vec{k} ; \vec{p}^{\prime} ; t, t^{\prime}\right) \cdot \frac{\partial}{\partial \vec{p}^{\prime}} \\
\quad+\frac{\mathrm{i} \vec{k}}{m} \cdot \vec{p} \varphi_{n F}\left(\vec{k} ; \vec{p}, \vec{p}^{\prime} ; t, t^{\prime}\right) \cdot \frac{\partial}{\partial \vec{p}^{\prime}}-\frac{\vec{k}}{m} \cdot \varphi_{j n}\left(\vec{k} ; \vec{p}, \vec{p}^{\prime} ; t, t^{\prime}\right) \frac{\vec{k}}{m} \cdot \vec{p}^{\prime}-\frac{\partial}{\partial \vec{p}} \cdot \vec{p} \varphi_{F n}\left(\vec{k} ; \vec{p}, \vec{p}^{\prime} ; t, t^{\prime}\right) \frac{\mathrm{i} \vec{k}}{m} \cdot \vec{p}^{\prime} \\
\quad-\frac{\beta}{m} \vec{p} \cdot \vec{\Phi}_{F h}(\vec{k}) f_{0}(p) \varphi_{h n}\left(\vec{k} ; \vec{p}^{\prime} ; t, t^{\prime}\right) \frac{\mathrm{i} \vec{k}}{m} \cdot \vec{p}^{\prime}+\frac{\vec{k}}{m} \cdot \vec{p} \varphi_{n n}^{(0)}\left(\vec{k} ; \vec{p}^{\prime} ; t, t^{\prime}\right) \frac{\vec{k}}{m} \cdot \vec{p}^{\prime},
\end{aligned}
$$

and contains the following time correlation functions

$$
\begin{aligned}
\varphi_{n n}^{(0)}\left(\vec{k} ; \vec{p}, \vec{p}^{\prime} ; t, t^{\prime}\right) & =\left\langle\hat{n}_{\vec{k}}(\vec{p}) T_{0}\left(t, t^{\prime}\right) \hat{n}_{-\vec{k}}\left(\vec{p}^{\prime}\right)\right\rangle_{0}, & & \varphi_{h n}\left(\vec{k} ; \vec{p}^{\prime} ; t, t^{\prime}\right)=\left\langle\hat{h}_{\vec{k}}^{\mathrm{int}} T_{0}\left(t, t^{\prime}\right) \hat{n}_{-\vec{k}}\left(\vec{p}^{\prime}\right)\right\rangle_{0}, \\
\varphi_{h \jmath}\left(\vec{k} ; \vec{p}^{\prime} ; t, t^{\prime}\right) & =\left\langle\hat{h}_{\vec{k}}^{\mathrm{int}} T_{0}\left(t, t^{\prime}\right) \hat{\vec{\jmath}}_{-\vec{k}}\left(\vec{p}^{\prime}\right)\right\rangle_{0}, & & \varphi_{h F}\left(\vec{k} ; \vec{p}^{\prime} ; t, t^{\prime}\right)=\left\langle\hat{h}_{\vec{k}}^{\mathrm{int}} T_{0}\left(t, t^{\prime}\right) \hat{\vec{F}}_{-\vec{k}}\left(\vec{p}^{\prime}\right)\right\rangle_{0}, \\
\varphi_{n \jmath}\left(\vec{k} ; \vec{p}, \vec{p}^{\prime} ; t, t^{\prime}\right) & =\left\langle\hat{n}_{\vec{k}}(\vec{p}) T_{0}\left(t, t^{\prime}\right) \hat{\vec{\jmath}}_{-\vec{k}}\left(\vec{p}^{\prime}\right)\right\rangle_{0}, & & \varphi_{n F}\left(\vec{k} ; \vec{p}, \vec{p}^{\prime} ; t, t^{\prime}\right)=\left\langle\hat{n}_{\vec{k}}(\vec{p}) T_{0}\left(t, t^{\prime}\right) \hat{\vec{F}}_{-\vec{k}}\left(\vec{p}^{\prime}\right)\right\rangle_{0}, \\
\varphi_{F n}\left(\vec{k} ; \vec{p}, \vec{p}^{\prime} ; t, t^{\prime}\right) & =\left\langle\hat{\vec{F}}_{\vec{k}}(\vec{p}) T_{0}\left(t, t^{\prime}\right) \hat{n}_{-\vec{k}}\left(\vec{p}^{\prime}\right)\right\rangle_{0}, & & \varphi_{n h}\left(\vec{k} ; \vec{p}^{\prime} ; t, t^{\prime}\right)=\left\langle\hat{n}_{\vec{k}}\left(\vec{p}^{\prime}\right) T_{0}\left(t, t^{\prime}\right) \hat{h}_{-\vec{k}}^{\text {int }}\right\rangle_{0},
\end{aligned}
$$

constructed on the basic set of dynamic variables $\hat{n}_{\vec{k}}(\vec{p}), \hat{h}_{\vec{k}}^{\text {int }}$ along with the Fourier-components of the momentum density $\hat{\vec{\jmath}}_{\vec{k}}(\vec{p})$ and the force $\vec{F}_{\vec{k}}(\vec{p})$ in momentum space. Moreover, $\hat{n}_{\vec{k}}(\vec{p}), \hat{\vec{\jmath}}_{\vec{k}}(\vec{p})$ and 
$\vec{F}_{\vec{k}}(\vec{p})$ are connected by the equation of motion (34). In this respect we assume $T_{0}\left(t, t^{\prime}\right)=\mathrm{e}^{\mathrm{i} L_{N}\left(t^{\prime}-t\right)}$. Then (47) represents the time correlation functions whose evolution does not contain projection.

The time correlation functions (47) enter $\varphi_{n j}^{(2)}\left(\vec{k} ; \vec{p} ; t, t^{\prime}\right)$ as well:

$$
\begin{aligned}
\varphi_{n \jmath}^{(2)}\left(\vec{k} ; \vec{p} ; t, t^{\prime}\right)= & \frac{\mathrm{i} \vec{k}}{m} \cdot \vec{p} \varphi_{n h}\left(\vec{k} ; \vec{p} ; t, t^{\prime}\right) \frac{\beta}{m n} \vec{\Phi}_{F h}(\vec{k})+\frac{\beta}{m} \vec{p} \cdot \vec{\Phi}_{F h}(\vec{k}) f_{0}(p) \varphi_{h h}\left(\vec{k} ; t, t^{\prime}\right) \frac{\beta}{m n} \vec{\Phi}_{F h}(\vec{k}) \\
& -\frac{\mathrm{i} \vec{k}}{m} \cdot \varphi_{\jmath h}\left(\vec{k} ; \vec{p} ; t, t^{\prime}\right) \frac{\beta}{m n} \vec{\Phi}_{F h}(\vec{k})+\frac{\partial}{\partial \vec{p}} \cdot \varphi_{F h}\left(\vec{k} ; \vec{p} ; t, t^{\prime}\right) \frac{\beta}{m n} \vec{\Phi}_{F h}(\vec{k}),
\end{aligned}
$$

where

$$
\begin{aligned}
& \varphi_{\jmath h}\left(\vec{k} ; \vec{p} ; t, t^{\prime}\right)=\left\langle\hat{\vec{\jmath}}_{\vec{k}}(\vec{p}) T_{0}\left(t, t^{\prime}\right) \hat{h}_{-\vec{k}}^{\mathrm{int}}\right\rangle_{0}, \quad \varphi_{F h}\left(\vec{k} ; \vec{p} ; t, t^{\prime}\right)=\left\langle\vec{F}_{\vec{k}}(\vec{p}) T_{0}\left(t, t^{\prime}\right) \hat{h}_{-\vec{k}}^{\mathrm{int}}\right\rangle_{0}, \\
& \varphi_{h h}\left(\vec{k} ; t, t^{\prime}\right)=\left\langle\hat{h}_{\vec{k}}^{\mathrm{int}} T_{0}\left(t, t^{\prime}\right) \hat{h}_{-\vec{k}}^{\mathrm{int}}\right\rangle_{0}
\end{aligned}
$$

the time correlation functions of the Fourier-components of densities of the potential part of enthalpy, momentum $\hat{\vec{j}}_{\vec{k}}(\vec{p})$ and force $\vec{F}_{\vec{k}}(\vec{p})$ in impulse space.

In the kinetic equation (27) the transport kernel $\varphi_{n h}\left(\vec{k} ; \vec{p} ; t, t^{\prime}\right)$ describes dynamic correlations between the kinetic and hydrodynamic processes. Performing the action of operators $\left(1-P_{0}\right)$ and $\mathrm{i} L_{N}$ as well as taking into account (37) and

$$
P_{0} \dot{\hat{h}}_{\vec{k}}^{\mathrm{int}}={\overrightarrow{\Phi^{\prime}}}_{h F}(\vec{k}) \cdot \hat{\vec{\jmath}}_{\vec{k}}, \quad{\overrightarrow{\Phi^{\prime}}}_{h F}(\vec{k})=\left\langle\hat{h}_{\vec{k}}^{\mathrm{int}} \vec{F}_{-\vec{k}}\right\rangle_{0} \frac{\beta}{m n},
$$

the kernel $\varphi_{n h}\left(\vec{k} ; \vec{p} ; t, t^{\prime}\right)$ can be presented as

$$
\begin{aligned}
& \varphi_{n h}\left(\vec{k} ; \vec{p} ; t, t^{\prime}\right)=-\frac{\mathrm{i} \vec{k}}{m} \cdot \bar{\varphi}_{\jmath \dot{h}}\left(\vec{k} ; \vec{p} ; t, t^{\prime}\right)+\frac{\partial}{\partial \vec{p}} \cdot \bar{\varphi}_{F \dot{h}}\left(\vec{k} ; \vec{p} ; t, t^{\prime}\right)+\frac{\beta}{m} \vec{p} \cdot \vec{\Phi}_{F h}(\vec{k}) f_{0}(p) \bar{\varphi}_{h \dot{h}}\left(\vec{k} ; t, t^{\prime}\right) \\
& \quad+\frac{\mathrm{i} \vec{k}}{m} \cdot \vec{p} \bar{\varphi}_{n \dot{h}}\left(\vec{k} ; \vec{p} ; t, t^{\prime}\right)+\frac{\mathrm{i} \vec{k}}{m} \cdot \varphi_{\jmath \jmath}\left(\vec{k} ; \vec{p} ; t, t^{\prime}\right) \cdot \vec{\Phi}_{h F}^{\prime}(\vec{k})-\frac{\partial}{\partial \vec{p}} \cdot \varphi_{F \jmath}\left(\vec{k} ; \vec{p} ; t, t^{\prime}\right) \vec{\Phi}_{h F}^{\prime}(\vec{F}) \\
& \quad-\frac{\beta}{m} \vec{p} \cdot \vec{\Phi}_{F h}(\vec{k}) f_{0}(p) \varphi_{h \jmath}\left(\vec{k} ; t, t^{\prime}\right) \cdot \vec{\Phi}_{h F}^{\prime}(\vec{k})-\frac{\mathrm{i} \vec{k}}{m} \cdot \vec{p} \varphi_{n \jmath}\left(\vec{k} ; \vec{p} ; t, t^{\prime}\right) \vec{\Phi}_{h F}^{\prime}(\vec{k})
\end{aligned}
$$

where

$$
\begin{aligned}
& \bar{\varphi}_{\jmath \dot{h}}\left(\vec{k} ; \vec{p} ; t, t^{\prime}\right)=\left\langle\hat{\vec{j}}_{\vec{k}}(\vec{p}) T_{0}\left(t, t^{\prime}\right) \dot{\hat{h}}_{-\vec{k}}^{\mathrm{int}}\right\rangle_{0} \Phi_{h h}^{-1}(\vec{k}), \bar{\varphi}_{F \dot{h}}\left(\vec{k} ; \vec{p} ; t, t^{\prime}\right)=\left\langle\vec{F}_{\vec{k}}(\vec{p}) T_{0}\left(t, t^{\prime}\right) \dot{\hat{h}}{ }_{-\vec{k}}^{\mathrm{int}}\right\rangle_{0} \Phi_{h h}^{-1}(\vec{k}), \\
& \bar{\varphi}_{h \dot{h}}\left(\vec{k} ; t, t^{\prime}\right)=\left\langle\hat{h}_{\vec{k}}^{\mathrm{int}}(\vec{p}) T_{0}\left(t, t^{\prime}\right) \dot{\hat{h}}_{-\vec{k}}^{\mathrm{int}}\right\rangle_{0} \Phi_{h h}^{-1}(\vec{k}), \\
& \bar{\varphi}_{n \dot{h}}\left(\vec{k} ; t, t^{\prime}\right)=-\left\langle\dot{\hat{n}}_{\vec{k}}(\vec{p}) T_{0}\left(t, t^{\prime}\right) \hat{h}_{-\vec{k}}^{\mathrm{int}}\right\rangle_{0} \Phi_{h h}^{-1}(\vec{k})=\frac{\mathrm{i} \vec{k}}{m} \cdot \bar{\varphi}_{\jmath h}\left(\vec{k} ; \vec{p} ; t, t^{\prime}\right)-\frac{\partial}{\partial \vec{p}} \cdot \bar{\varphi}_{F h}\left(\vec{k} ; \vec{p} ; t, t^{\prime}\right), \\
& \bar{\varphi}_{\jmath h}\left(\vec{k} ; \vec{p} ; t, t^{\prime}\right)=\varphi_{\jmath h}\left(\vec{k} ; \vec{p} ; t, t^{\prime}\right) \Phi_{h h}^{-1}(\vec{k}), \quad \bar{\varphi}_{F h}\left(\vec{k} ; \vec{p} ; t, t^{\prime}\right)=\varphi_{F h}\left(\vec{k} ; \vec{p} ; t, t^{\prime}\right) \Phi_{h h}^{-1}(\vec{k})
\end{aligned}
$$

are the normalized time correlation functions. The correlation functions $\varphi_{\jmath \jmath}\left(\vec{k} ; \vec{p} ; t, t^{\prime}\right), \varphi_{F \jmath}\left(\vec{k} ; \vec{p} ; t, t^{\prime}\right)$, $\varphi_{h \jmath}\left(\vec{k} ; \vec{p} ; t, t^{\prime}\right)$, and $\varphi_{n \jmath}\left(\vec{k} ; \vec{p} ; t, t^{\prime}\right)$ have the structure similar to (47). From the structure of transport kernels (41), (42), (46), (48) and (50) in the kinetic equation (27) for the nonequilibrium one-particle distribution function one can see that the contributions of hydrodynamic processes are described, besides $\hat{h}_{\vec{k}}^{\text {int }}$, by the moments $\int \mathrm{d} \vec{p} f_{\vec{k}}(\vec{p} ; t)=n_{\vec{k}}(t)=\left\langle\hat{n}_{\vec{k}}\right\rangle^{t}, \int \mathrm{d} \vec{p} \vec{p} f_{\vec{k}}(\vec{p} ; t)=\left\langle\hat{\vec{j}}_{\vec{k}}\right\rangle^{t}$. As in the case of equation (27), let us find the inner structure of transport kernels in equation (28) for the average value of the potential part of enthalpy. In particular, taking into account (50), for $\varphi_{h h}\left(\vec{k} ; t, t^{\prime}\right)$ we obtain:

$$
\begin{aligned}
\varphi_{h h}\left(\vec{k} ; t, t^{\prime}\right)= & \bar{\varphi}_{\dot{h} \dot{h}}^{(0)}\left(\vec{k} ; t, t^{\prime}\right)-\vec{\Phi}_{h F}^{\prime}(\vec{k}) \cdot \bar{\varphi}_{j \dot{h}}^{(0)}\left(\vec{k} ; t, t^{\prime}\right) \\
& -\bar{\varphi}_{\dot{h}_{\jmath}}\left(\vec{k} ; t, t^{\prime}\right) \cdot \vec{\Phi}_{h F}^{\prime}(\vec{k})+\vec{\Phi}_{h F}^{\prime}(\vec{k}) \cdot \varphi_{\jmath \jmath}^{(0)}\left(\vec{k} ; t, t^{\prime}\right) \cdot \vec{\Phi}_{F h}(\vec{k}) .
\end{aligned}
$$




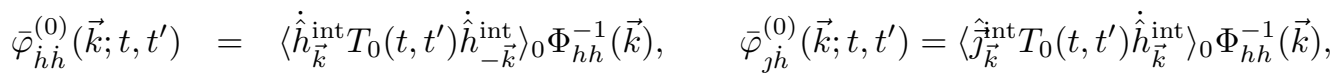

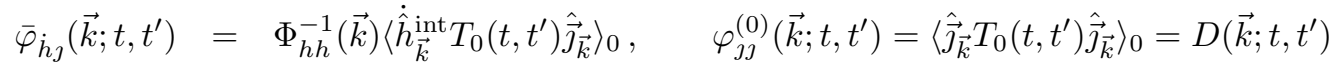

is the generalized diffusion coefficient (44). Taking into account (37), (51) the transport kernel $\varphi_{h n}\left(\vec{k} ; \vec{p} ; t, t^{\prime}\right)$ entering (28) as $\int \mathrm{d} \vec{p}^{\prime} \varphi_{h n}\left(\vec{k} ; \vec{p}^{\prime} ; t, t^{\prime}\right) f_{\vec{k}}\left(\vec{p}^{\prime} ; t^{\prime}\right)$ can be presented in the following way

$$
\begin{aligned}
& \int \mathrm{d} \vec{p}^{\prime} \varphi_{h n}\left(\vec{k} ; \vec{p}^{\prime} ; t, t^{\prime}\right) f_{\vec{k}}\left(\vec{p}^{\prime} ; t^{\prime}\right)=\frac{\mathrm{i} \vec{k}}{m} \cdot \int \mathrm{d} \vec{p}^{\prime} W_{h n}\left(\vec{k} ; \vec{p}^{\prime} ; t, t^{\prime}\right) \frac{1}{n f_{0}\left(p^{\prime}\right)} f_{\vec{k}}\left(\vec{p}^{\prime} ; t\right) \\
& \quad-\int \mathrm{d} \vec{p}^{\prime} W_{h F}\left(\vec{k} ; \vec{p}^{\prime} ; t, t^{\prime}\right) \frac{1}{n f_{0}\left(p^{\prime}\right)} \cdot\left(\beta \frac{\vec{p}^{\prime}}{m n}-\frac{\partial}{\partial \vec{p}^{\prime}}\right) f_{\vec{k}}\left(\vec{p}^{\prime} ; t^{\prime}\right)+W_{h j}\left(\vec{k} ; t, t^{\prime}\right) \frac{\beta}{m n} \cdot\left\langle\hat{\vec{\jmath}} \vec{k} t^{\prime},\right.
\end{aligned}
$$

where

$$
\begin{aligned}
W_{h n}\left(\vec{k} ; \vec{p}^{\prime} ; t, t^{\prime}\right)= & \varphi_{h_{\jmath}}\left(\vec{k} ; \vec{p}^{\prime} ; t, t^{\prime}\right)-\varphi_{h n}\left(\vec{k} ; \vec{p}^{\prime} ; t, t^{\prime}\right) \cdot \vec{p}^{\prime} \\
& -\vec{\Phi}_{h F}^{\prime}(\vec{k}) \varphi_{\jmath \jmath}\left(\vec{k} ; \vec{p}^{\prime} ; t, t^{\prime}\right)+\vec{\Phi}_{h F}^{\prime}(\vec{k}) \varphi_{\jmath n}\left(\vec{k} ; \vec{p}^{\prime} ; t, t^{\prime}\right) \cdot \vec{p}^{\prime}, \\
W_{h F}\left(\vec{k} ; \vec{p}^{\prime} ; t, t^{\prime}\right)= & \varphi_{h F}\left(\vec{k} ; \vec{p}^{\prime} ; t, t^{\prime}\right)+\vec{\Phi}_{h F}^{\prime}(\vec{k}) \varphi_{\jmath F}\left(\vec{k} ; \vec{p}^{\prime} ; t, t^{\prime}\right), \\
W_{h \jmath}\left(\vec{k} ; t, t^{\prime}\right)= & \varphi_{h h}\left(\vec{k} ; t, t^{\prime}\right) \vec{\Phi}_{h F}^{\prime}(\vec{k})-\vec{\Phi}_{h F}^{\prime}(\vec{k}) \varphi_{\jmath h}\left(\vec{k} ; t, t^{\prime}\right) \vec{\Phi}_{h F}^{\prime}(\vec{k})
\end{aligned}
$$

are the transport kernels, formed by the time correlation functions of the type of (52), (153) and (55). Taking into account the structure of the memory functions (41), (51), (54) and (57), we present the set of equations (27), (28) in the form

$$
\begin{aligned}
& \frac{\partial}{\partial t} f_{\vec{k}}(\vec{p} ; t)+\frac{\mathrm{i} \vec{k}}{m} \cdot \vec{p} f_{\vec{k}}(\vec{p} ; t)=-\frac{\mathrm{i} \vec{k}}{m} \cdot \vec{p} n f_{0}(p) c_{2}(\vec{k}) \int \mathrm{d} \vec{p}^{\prime} f_{\vec{k}}\left(\vec{p}^{\prime} ; t\right)+\mathrm{i} \Omega_{n h}(\vec{k} ; \vec{p}) h_{\vec{k}}^{\operatorname{int}}(t) \\
& -\int \mathrm{d} \vec{p}^{\prime} \int_{-\infty}^{t} \mathrm{e}^{\varepsilon\left(t^{\prime}-t\right)}\left\{\varphi_{\jmath \jmath}\left(\vec{k} ; \vec{p}, \vec{p}^{\prime} ; t, t^{\prime}\right)\right. \\
& \left.-\frac{\partial}{\partial \vec{p}} \cdot \varphi_{F F}\left(\vec{k} ; \vec{p}, \vec{p}^{\prime} ; t, t^{\prime}\right) \cdot\left(\frac{\beta \vec{p}^{\prime}}{m n f_{0}\left(p^{\prime}\right)}-\frac{\partial}{\partial \vec{p}^{\prime}}\right)\right\} f_{\vec{k}}\left(\vec{p}^{\prime} ; t^{\prime}\right) \mathrm{d} t^{\prime} \\
& +\int_{-\infty}^{t} \mathrm{e}^{\varepsilon\left(t^{\prime}-t\right)} \varphi_{n j}^{(2)}\left(\vec{k} ; \vec{p}, ; t, t^{\prime}\right) \cdot \vec{\jmath}_{\vec{k}}(t) \mathrm{d} t^{\prime}+\int_{-\infty}^{t} \mathrm{e}^{\varepsilon\left(t^{\prime}-t\right)}\left\{\frac{\mathrm{i} \vec{k}}{m} \cdot W_{n \dot{h}}\left(\vec{k} ; \vec{p} ; t, t^{\prime}\right)-\frac{\partial}{\partial \vec{p}} \cdot W_{F h}\left(\vec{k} ; \vec{p} ; t, t^{\prime}\right)\right. \\
& \left.-\frac{\beta}{m} f_{0}(p) \vec{p} \cdot W_{F \dot{h}}\left(\vec{k} ; \vec{p} ; t, t^{\prime}\right)\right\} h_{\vec{k}}^{\mathrm{int}}\left(t^{\prime}\right) \mathrm{d} t^{\prime} \\
& \frac{\partial}{\partial t} h_{\vec{k}}^{\text {int }}(t)=\int \mathrm{d} \vec{p}^{\prime} \mathrm{i} \Omega_{h n}\left(\vec{k} ; \vec{p}^{\prime}\right) f_{\vec{k}}\left(\vec{p}^{\prime} ; t\right)-\int_{-\infty}^{t} \mathrm{e}^{\varepsilon\left(t^{\prime}-t\right)}\left\{\bar{\varphi}_{\dot{h} \dot{h}}^{(0)}\left(\vec{k} ; t, t^{\prime}\right)-\vec{\Phi}_{h F}^{\prime}(\vec{k}) \cdot \bar{\varphi}_{j h}^{(0)}\left(\vec{k} ; t, t^{\prime}\right)\right. \\
& \left.-\bar{\varphi}_{h \jmath}\left(\vec{k} ; t, t^{\prime}\right) \cdot \vec{\Phi}_{h F}^{\prime}(\vec{k})+\vec{\Phi}_{h F}^{\prime}(\vec{k}) \cdot \bar{\varphi}_{J \jmath}^{(0)}\left(\vec{k} ; t, t^{\prime}\right) \cdot \vec{\Phi}_{F h}(\vec{k})\right\} h_{\vec{k}}^{\mathrm{int}}\left(t^{\prime}\right) \mathrm{d} t^{\prime} \\
& -\frac{\mathrm{i} \vec{k}}{m} \cdot \int \mathrm{d} \vec{p}^{\prime} \int_{-\infty}^{t} \mathrm{e}^{\varepsilon\left(t^{\prime}-t\right)} W_{h n}\left(\vec{k} ; \vec{p}^{\prime} ; t, t^{\prime}\right) \frac{1}{n f_{0}\left(p^{\prime}\right)} f_{\vec{k}}\left(\vec{p}^{\prime} ; t^{\prime}\right) \mathrm{d} t^{\prime} \\
& +\int \mathrm{d} \vec{p}^{\prime} \int_{-\infty}^{t} \mathrm{e}^{\varepsilon\left(t^{\prime}-t\right)} W_{h F}\left(\vec{k} ; \vec{p}^{\prime} ; t, t^{\prime}\right) \frac{1}{n f_{0}\left(p^{\prime}\right)} \cdot\left(\frac{\beta}{m n} \vec{p}^{\prime}-\frac{\partial}{\partial \vec{p}^{\prime}}\right) f_{\vec{k}}\left(\vec{p}^{\prime} ; t^{\prime}\right) \mathrm{d} t^{\prime} \\
& -\int_{-\infty}^{t} \mathrm{e}^{\varepsilon\left(t^{\prime}-t\right)} W_{h_{\jmath}}\left(\vec{k} ; t, t^{\prime}\right) \frac{\beta}{m n} \cdot \vec{\jmath}_{\vec{k}}\left(t^{\prime}\right) \mathrm{d} t^{\prime},
\end{aligned}
$$


where in the first equation the transport kernels have the following structure:

$$
\begin{aligned}
W_{n \dot{h}}\left(\vec{k} ; \vec{p} ; t, t^{\prime}\right)= & \bar{\varphi}_{\jmath \dot{h}}\left(\vec{k} ; \vec{p} ; t, t^{\prime}\right)-\vec{p} \cdot \bar{\varphi}_{n \dot{h}}\left(\vec{k} ; \vec{p} ; t, t^{\prime}\right) \\
& -\varphi_{\jmath \jmath}\left(\vec{k} ; \vec{p} ; t, t^{\prime}\right) \cdot \vec{\Phi}_{h F}^{\prime}(\vec{k})+\vec{p} \cdot \varphi_{n \jmath}\left(\vec{k} ; \vec{p} ; t, t^{\prime}\right) \cdot \vec{\Phi}_{h F}^{\prime}(\vec{k}), \\
W_{F h}\left(\vec{k} ; \vec{p} ; t, t^{\prime}\right)= & \bar{\varphi}_{F h}\left(\vec{k} ; \vec{p} ; t, t^{\prime}\right)-\varphi_{F \jmath}\left(\vec{k} ; \vec{p} ; t, t^{\prime}\right) \cdot \vec{\Phi}_{h F}^{\prime}(\vec{k}), \\
W_{F h}\left(\vec{k} ; \vec{p} ; t, t^{\prime}\right)= & \vec{\Phi}_{F h}(\vec{k}) \cdot \varphi_{h \dot{h}}\left(\vec{k} ; t, t^{\prime}\right)-\vec{\Phi}_{F h}(\vec{k}) \cdot \varphi_{h \jmath}\left(\vec{k} ; t, t^{\prime}\right) \cdot \vec{\Phi}_{h F}^{\prime}(\vec{k}) .
\end{aligned}
$$

The transport equations (58), (59) are functionally connected concerning the basic parameters of reduced description $f_{\vec{k}}(\vec{p} ; t), h_{\vec{k}}^{\text {int }}(t)$. However, the equations contain the average values of densities of particles number $\left\langle\hat{n}_{\vec{k}}\right\rangle^{t}$ and momentum $\left\langle\hat{\vec{j}}_{\vec{k}}\right\rangle^{t}$, which, generally speaking, are the hydrodynamic variables. Integrating the equation (58) over momentum, we obtain the equation for $n_{\vec{k}}(t)$

$$
\frac{\partial}{\partial t} n_{\vec{k}}(t)+\frac{\mathrm{i} \vec{k}}{m} \vec{\jmath}_{\vec{k}}(t)=0
$$

that represents the conservation law for an average value of number of particles. The transport equations (58), (59) for $f_{\vec{k}}(\vec{p} ; t)$ and $h_{\vec{k}}^{\text {int }}(t)$ are written in $\{\vec{k}, \vec{p}, t\}$ space. According to (42), (47), (48), $f_{\vec{k}}(\vec{p} ; t)$ and $h_{\vec{k}}^{\text {int }}(t)$ are determined in terms of the time correlation functions of the basic set $\hat{n}_{\vec{k}}(\vec{p}), \hat{h}_{\vec{k}}^{\mathrm{int}}$ (47), (48). This means that the system of equation (58), (59) should be complemented by the equations for the appropriate time correlation functions of dynamic variables $\hat{n}_{\vec{k}}(\vec{p}), \hat{\vec{j}}_{\vec{k}}(\vec{p})$, $\vec{F}_{\vec{k}}(\vec{p})$ and $\hat{h}_{\vec{k}}^{\text {int }}$.

In [9], on the basis of the equations (58) and (59) there has been obtained the following set of transport equations for the time correlation functions:

$$
\begin{array}{ll}
\Phi_{n n}\left(\vec{k} ; \vec{p}, \vec{p}^{\prime} ; t\right)=\left\langle\hat{n}_{\vec{k}}(\vec{p} ; t) \hat{n}_{-\vec{k}}\left(\vec{p}^{\prime} ; 0\right)\right\rangle_{0}, & \Phi_{h n}^{\mathrm{int}}\left(\vec{k} ; \vec{p}^{\prime} ; t\right)=\left\langle\hat{h}_{\vec{k}}^{\mathrm{int}}(t) \hat{n}_{-\vec{k}}\left(\vec{p}^{\prime} ; 0\right)\right\rangle_{0}, \\
\Phi_{n h}^{\mathrm{int}}(\vec{k} ; \vec{p} ; t)=\left\langle\hat{n}_{\vec{k}}(\vec{p} ; t) \hat{h}_{-\vec{k}}^{\mathrm{int}}(0)\right\rangle_{0}, & \Phi_{h h}^{\mathrm{int}, \mathrm{int}}(\vec{k} ; t)=\left\langle\hat{h}_{\vec{k}}^{\mathrm{int}}(t) \hat{h}_{-\vec{k}}^{\mathrm{int}}(0)\right\rangle_{0} .
\end{array}
$$

Using the Laplace transform $A(z)=\mathrm{i} \int_{0}^{\infty} \mathrm{d} t \mathrm{e}^{\mathrm{i} z t} A(t)$, let us represent it in a matrix form,

$$
z \tilde{\Phi}(\vec{k} ; z)=\tilde{\Sigma}(\vec{k} ; z) \tilde{\Phi}(\vec{k} ; z)-\tilde{\Phi}(\vec{k})
$$

where $\hat{n}_{\vec{k}}(\vec{p} ; t)=\mathrm{e}^{-\mathrm{i} L_{N} t} \hat{n}_{\vec{k}}(\vec{p} ; 0), \hat{h}_{\vec{k}}^{\mathrm{int}}(t)=\mathrm{e}^{-\mathrm{i} L_{N} t} \hat{h}_{\vec{k}}^{\mathrm{int}}(0)$, and $\tilde{\Phi}(\vec{k} ; z)$ is the matrix whose elements are the Laplace transforms of the time correlation functions (61).

$$
\tilde{\Phi}(\vec{k})=\left(\begin{array}{cc}
\Phi_{n n}\left(\vec{k} ; \vec{p}, \vec{p}^{\prime}\right) & 0 \\
0 & \Phi_{h h}^{\text {int,int }}(\vec{k})
\end{array}\right), \quad \tilde{\Sigma}(\vec{k} ; z)=\left(\begin{array}{cc}
\int \mathrm{d} \vec{p}^{\prime \prime} \Sigma_{n n}\left(\vec{k} ; \vec{p}, \vec{p}^{\prime \prime} ; z\right) & \Sigma_{n h}(\vec{k} ; \vec{p} ; z) \\
\int \mathrm{d} \vec{p}^{\prime \prime} \Sigma_{h n}\left(\vec{k} ; \vec{p}^{\prime \prime} ; z\right) & -\varphi_{h h}(\vec{k} ; z)
\end{array}\right)
$$

where

$$
\begin{gathered}
\Sigma_{n n}\left(\vec{k} ; \vec{p}, \vec{p}^{\prime \prime} ; z\right)=\mathrm{i} \Omega_{n n}\left(\vec{k} ; \vec{p}, \vec{p}^{\prime \prime}\right)-\varphi_{n n}\left(\vec{k} ; \vec{p}, \vec{p}^{\prime \prime} ; z\right) \\
\Sigma_{n h}(\vec{k} ; \vec{p} ; z)=\mathrm{i} \Omega_{n h}(\vec{k} ; \vec{p})-\varphi_{n h}(\vec{k} ; \vec{p} ; z), \quad \Sigma_{h n}\left(\vec{k} ; \vec{p}^{\prime \prime} ; z\right)=\mathrm{i} \Omega_{h n}\left(\vec{k} ; \vec{p}^{\prime \prime}\right)-\varphi_{h n}\left(\vec{k} ; \vec{p}^{\prime \prime} ; z\right) .
\end{gathered}
$$

Taking into account the structure of memory functions (41), (51), (54) and (57), we present the set of equations (62) in the explicit form:

$$
\begin{aligned}
& z \Phi_{n n}\left(\vec{k} ; \vec{p}, \vec{p}^{\prime} ; z\right)+\frac{\mathrm{i} \vec{k} \cdot \vec{p}}{m} \Phi_{n n}\left(\vec{k} ; \vec{p}, \vec{p}^{\prime} ; z\right)= \\
& \quad-\frac{\mathrm{i} \vec{k} \cdot \vec{p}}{m} n f_{0}(p) c_{2}(k) \Phi_{n n}\left(\vec{k} ; \vec{p}, \vec{p}^{\prime} ; z\right)-\frac{\beta}{m} \vec{p} \cdot \vec{\Phi}_{F h}(\vec{k}) f_{0}(p) \Phi_{h n}^{\mathrm{int}}\left(\vec{k} ; \vec{p}^{\prime} ; z\right) \\
& \quad-\int \mathrm{d} \vec{p}^{\prime \prime}\left\{\tilde{\varphi}_{\jmath \jmath}\left(\vec{k} ; \vec{p}, \vec{p}^{\prime \prime} ; z\right)-\frac{\partial}{\partial \vec{p}} \varphi_{F F}\left(\vec{k} ; \vec{p}, \vec{p}^{\prime \prime} ; z\right)\left(\frac{\beta \vec{p}^{\prime \prime}}{m n f_{0}\left(p^{\prime \prime}\right)}-\frac{\partial}{\partial \vec{p}^{\prime \prime}}\right)\right\} \Phi_{n n}\left(\vec{k} ; \vec{p}^{\prime \prime}, \vec{p}^{\prime} ; z\right) \\
& \quad-\tilde{\varphi}_{n \jmath}^{(2)}(\vec{k} ; \vec{p} ; z) \Phi_{\jmath n}\left(\vec{k} ; \vec{p}^{\prime} ; z\right)-\varphi_{n h}(\vec{k} ; \vec{p} ; z) \Phi_{h n}^{\mathrm{int}}\left(\vec{k} ; \vec{p}^{\prime} ; z\right)-\Phi_{n n}\left(\vec{k} ; \vec{p}, \vec{p}^{\prime}\right),
\end{aligned}
$$




$$
\begin{aligned}
& z \Phi_{n h}^{\mathrm{int}}(\vec{k} ; \vec{p} ; z)+\frac{\mathrm{i} \vec{k} \cdot \vec{p}}{m} \Phi_{n h}^{\mathrm{int}}(\vec{k} ; \vec{p} ; z)= \\
& -\frac{\mathrm{i} \vec{k} \cdot \vec{p}}{m} n f_{0}(p) c_{2}(k) \Phi_{n h}^{\mathrm{int}}(\vec{k} ; \vec{p} ; z)-\frac{\beta}{m} \vec{p} \cdot \vec{\Phi}_{F h}(\vec{k}) f_{0}(p) \Phi_{h h}^{\mathrm{int}, \mathrm{int}}(\vec{k} ; z) \\
& -\int \mathrm{d} \vec{p}^{\prime \prime}\left\{\tilde{\varphi}_{J \jmath}\left(\vec{k} ; \vec{p}, \vec{p}^{\prime \prime} ; z\right)-\frac{\partial}{\partial \vec{p}} \varphi_{F F}\left(\vec{k} ; \vec{p}, \vec{p}^{\prime \prime} ; z\right)\left(\frac{\beta \vec{p}^{\prime \prime}}{m n f_{0}\left(p^{\prime \prime}\right)}-\frac{\partial}{\partial \vec{p}^{\prime \prime}}\right)\right\} \Phi_{n h}^{\mathrm{int}}\left(\vec{k} ; \vec{p}^{\prime \prime} ; z\right) \\
& -\tilde{\varphi}_{n \jmath}^{(2)}(\vec{k} ; \vec{p} ; z) \Phi_{\jmath h}^{\mathrm{int}}(\vec{k} ; z)-\varphi_{n h}(\vec{k} ; \vec{p} ; z) \Phi_{h h}^{\mathrm{int} \text { int }}(\vec{k} ; z), \\
& z \Phi_{h n}^{\operatorname{int}}\left(\vec{k} ; \vec{p}^{\prime} ; z\right)=-\int \mathrm{d} \vec{p}^{\prime \prime}\left\{\frac{\mathrm{i} \vec{k}}{m} \cdot W_{h n}\left(\vec{k} ; \vec{p}^{\prime \prime} ; z\right) \frac{1}{n f_{0}\left(p^{\prime \prime}\right)}\right. \\
& \left.-W_{h F}\left(\vec{k} ; \vec{p}^{\prime \prime} ; z\right) \frac{1}{n f_{0}\left(p^{\prime \prime}\right)}\left(\frac{\beta \vec{p}^{\prime \prime}}{m n}-\frac{\partial}{\partial \vec{p}^{\prime \prime}}\right)\right\} \Phi_{n n}\left(\vec{k} ; \vec{p}^{\prime \prime}, \vec{p}^{\prime} ; z\right) \\
& -W_{h \jmath}(\vec{k} ; z) \frac{\beta}{m n} \Phi_{\jmath n}\left(\vec{k} ; \vec{p}^{\prime} ; z\right)-\varphi_{h h}(\vec{k} ; z) \Phi_{h n}^{\mathrm{int}}\left(\vec{k} ; \vec{p}^{\prime} ; z\right), \\
& z \Phi_{h h}^{\text {int,int }}(\vec{k} ; z)=-\int \mathrm{d} \vec{p}^{\prime \prime}\left\{\frac{\mathrm{i} \vec{k}}{m} \cdot W_{h n}\left(\vec{k} ; \vec{p}^{\prime \prime} ; z\right) \frac{1}{n f_{0}\left(p^{\prime \prime}\right)}\right. \\
& \left.-W_{h F}\left(\vec{k} ; \vec{p}^{\prime \prime} ; z\right) \frac{1}{n f_{0}\left(p^{\prime \prime}\right)}\left(\frac{\beta \vec{p}^{\prime \prime}}{m n}-\frac{\partial}{\partial \vec{p}^{\prime \prime}}\right)\right\} \Phi_{n h}^{\mathrm{int}}\left(\vec{k} ; \vec{p}^{\prime} ; z\right) \\
& -W_{h \jmath}(\vec{k} ; z) \frac{\beta}{m n} \Phi_{\jmath h}^{\mathrm{int}}(\vec{k} ; z)-\varphi_{h h}(\vec{k} ; z) \Phi_{h h}^{\mathrm{int}, \text { int }}(\vec{k} ; z)-\Phi_{h h}^{\mathrm{int}, \text { int }}(\vec{k}) .
\end{aligned}
$$

The extended set of equations (58), (59) and (65)-(68) at fixed values of normalized correlation functions (29) and transport kernels (55) describing diffusive, viscous and heat-conduction processes $\left(\varphi_{\dot{h} h}^{(0)}\right.$ is the potential part of the generalized heat-conductivity coefficient) can be solved, in principle, in $\{\vec{k}, \vec{p}, t\}$ space with the help of numerical methods. Obviously, such solutions could provide interesting and important information, in particular, on the behavior of the time correlation function $\left\langle\hat{n}_{\vec{k}}(\vec{p} ; t) \hat{n}_{\vec{k}}\left(\vec{p}^{\prime} ; 0\right)\right\rangle_{0}$, on momentum. By integrating over momentum variables, this function can be connected with the time correlation function $\left\langle\hat{n}_{\vec{k}}(t) \hat{n}_{\vec{k}}(0)\right\rangle_{0}$ and, thus, with the dynamic structure factor of the system $S(\vec{k} ; \omega)$.

Projecting the set of equations (58), (59) onto the first moments of the nonequilibrium oneparticle distribution function $\Psi_{1}(\vec{p})=1, \Psi_{\alpha}(\vec{p})=\sqrt{2} p_{\alpha} / 2 k_{\mathrm{B}} T, \Psi_{\varepsilon}(\vec{p})=\sqrt{2 / 3}\left(p^{2} / 2 m k_{\mathrm{B}} T-3 / 2\right)$ $(\alpha=x, y, z)$ one can obtain the set of equations [9] for the averaged values of densities of particles number $n_{\vec{k}}(t)$, momentum $\vec{\jmath}_{\vec{k}}(t)$, kinetic $h_{\vec{k}}^{\text {kin }}(t)$ and potential $h_{\vec{k}}^{\text {int }}(t)$ parts of enthalpy. Similarly, projecting the set of equations (65)-(68) onto the same moments [9], we obtain a set of equations of the type of (62) for the appropriate time correlation functions

$$
\tilde{\Phi}(\vec{k} ; z)=\left(\begin{array}{llll}
\Phi_{n n} & \Phi_{n \jmath} & \Phi_{n h}^{\text {kin }} & \Phi_{n h}^{\text {int }} \\
\Phi_{\jmath n} & \Phi_{\jmath \jmath} & \Phi_{l h}^{\text {kin }} & \Phi_{l h}^{\text {int }} \\
\Phi_{h n}^{\text {kin }} & \Phi_{h \jmath}^{\text {kin }} & \Phi_{h h}^{\text {kin,kin }} & \Phi_{h h}^{\text {kint }} \\
\Phi_{h n}^{\text {int }} & \Phi_{h \jmath}^{\text {int }} & \Phi_{h h}^{\text {int }, \text { in }} & \Phi_{h h}^{\text {int,int }}
\end{array}\right)_{(\vec{k} ; z)}
$$

with the matrix of memory kernels $\tilde{\Sigma}_{G}(\vec{k} ; z)$.

$$
\tilde{\Sigma}_{G}(\vec{k} ; z)=\mathrm{i} \tilde{\Omega}_{G}(\vec{k})+\tilde{\Pi}(\vec{k} ; z)
$$

where

$$
\mathrm{i} \tilde{\Omega}(\vec{k})=\left(\begin{array}{llll}
0 & \mathrm{i} \Omega_{n \jmath} & 0 & 0 \\
\mathrm{i} \Omega_{\jmath n} & 0 & \mathrm{i} \Omega_{\jmath h}^{\mathrm{kin}} & \mathrm{i} \Omega_{\jmath h}^{\mathrm{int}} \\
0 & \mathrm{i} \Omega_{h \jmath}^{\mathrm{kin}} & 0 & 0 \\
0 & \mathrm{i} \Omega_{h \jmath}^{\mathrm{int}} & 0 & 0
\end{array}\right)_{(\vec{k})}
$$


is the frequency matrix, $\hat{h}_{\vec{k}}^{\text {kin }}=\hat{\varepsilon}_{\vec{k}}^{\text {kin }}-\left\langle\hat{\varepsilon}_{\vec{k}}^{\mathrm{kin}} \hat{n}_{-\vec{k}}\right\rangle_{0}\left\langle\hat{n}_{\vec{k}} \hat{n}_{-\vec{k}}\right\rangle_{0}^{-1} \hat{n}_{\vec{k}}$ are the Fourier-components of the kinetic part of enthalpy density.

$$
\tilde{\Pi}(\vec{k} ; z)=\left(\begin{array}{llll}
0 & 0 & 0 & 0 \\
0 & \Pi_{\jmath \jmath} & \Pi_{\gamma_{h}^{\mathrm{kin}}}^{\mathrm{kin}} & \Pi_{\gamma_{h}^{\mathrm{int}}} \\
0 & \Pi_{h \jmath}^{\text {kin }} & \Pi_{h h}^{\text {kin,kin }} & \Pi_{h h}^{\text {kin, int }} \\
0 & \Pi_{h \jmath}^{\text {int }} & \Pi_{h h}^{\text {int }, k i n} & \Pi_{h h}^{\text {int,int }}
\end{array}\right)_{(\vec{k} ; z)}
$$

is the matrix of transport kernels. Its elements have the following structure:

$$
\Pi_{\mu \nu}(\vec{k} ; z)=\left\langle\Psi_{\mu}\left|\tilde{\varphi}(\vec{k} ; z)+\tilde{\Sigma}(\vec{k} ; z) \mathcal{Q}[z \tilde{I} \mathcal{Q} \tilde{\Sigma}(\vec{k} ; z) \mathcal{Q}]^{-1} \mathcal{Q} \tilde{\Sigma}(\vec{k} ; z)\right| \Psi_{\nu}\right\rangle,
$$

where $\mathcal{Q}=1-\mathcal{P} . \mathcal{P}$ is the projection operator constructed on the eigenfunctions $\left|\Psi_{\alpha}(\vec{p})\right\rangle$ of the nonequilibrium one-particle function and $\mathcal{P}\langle\Psi|=\sum_{\nu=1}^{n}\left\langle\Psi \mid \Psi_{\nu}\right\rangle\left\langle\Psi_{\nu}\right| \cdot\left\langle\Psi \mid \Psi_{\nu}\right\rangle=\int \mathrm{d} \vec{\xi} \Psi(\vec{\xi}) f_{0}(\xi) \Psi_{\nu}(\vec{\xi})$, while $\Psi_{\nu}(\xi)$ satisfy the conditions $\left\langle\Psi_{\mu} \mid \Psi_{\nu}\right\rangle=\delta_{\mu \nu}, \sum_{\nu}\left|\Psi_{\nu}\right\rangle\left\langle\Psi_{\nu}\right|=1$. As we can see from the structure of elements of the matrixes $i \tilde{\Omega}_{G}(\vec{k})$ and $\tilde{\Pi}(k ; z)$, the contributions of kinetic and potential parts of enthalpy are separated. However, all the transport kernels of $\tilde{\Pi}(k ; \vec{z})$ are determined in terms of the time correlation functions (47), (49) and the transport kernels $\bar{\varphi}_{\dot{h} \dot{h}}^{(0)}\left(\vec{k} ; t, t^{\prime}\right), \bar{\varphi}_{j h}^{(0)}\left(\vec{k} ; t, t^{\prime}\right)$, $\bar{\varphi}_{h_{\jmath}}^{(0)}\left(\vec{k} ; t, t^{\prime}\right)$ and $D\left(\vec{k} ; t, t^{\prime}\right)$ (55). Herewith, a question arises regarding the study of time correlation functions (69) and collective modes for liquids using the generalized collective modes approach [27, 28].

We note one more important feature of the system (27)-(28). Let us suppose particles interact through the potential presented as

$$
\Phi\left(\left|\vec{r}_{i j}\right|\right)=\Phi^{h s}\left(\left|\vec{r}_{i j}\right|\right)+\Phi^{l}\left(\left|\vec{r}_{i j}\right|\right),
$$

where $\Phi^{h s}\left(\left|\vec{r}_{i j}\right|\right)$ is the hard sphere interaction potential, and $\Phi^{l}\left(\left|\vec{r}_{i j}\right|\right)$ is the long-range part of the potential. Taking into account the features of the hard sphere model dynamics [4] and investigations [20, 23, 25], one can separate Enskog-Boltzmann collision integral from the function $\varphi_{n n}\left(\vec{k} ; \vec{p}, \vec{p}^{\prime} ; t, t^{\prime}\right)$. Then the equation (27) can be written in the form

$$
\begin{aligned}
& \frac{\partial}{\partial t} f_{\vec{k}}(\vec{p} ; t)+\frac{\mathrm{i} \vec{k} \cdot \vec{p}}{m} f_{\vec{k}}(\vec{p} ; t)=-\frac{\mathrm{i} \vec{k} \cdot \vec{p}}{m} n f_{0}(\vec{p})\left(c_{2}(k)-g_{2}(\sigma) c_{2}^{0}(k)\right) \int \mathrm{d} \vec{p}^{\prime} f_{\vec{k}}\left(\vec{p}^{\prime} ; t\right) \\
& \quad-n g_{2}(\sigma) \sigma^{2} \int \mathrm{d} \Omega_{\sigma} \int \mathrm{d} \vec{p}_{1} \frac{\left(\vec{p}-\vec{p}_{1}\right) \cdot \hat{\vec{\sigma}}}{m} \Theta_{-}\left(\hat{\vec{\sigma}} \cdot\left(\vec{p}-\vec{p}_{1}\right)\right) \\
& \quad \times\left[f_{0}\left(p_{1}^{*}\right) f_{\vec{k}}(\vec{p} ; t)-f_{0}\left(p_{1}\right) f_{\vec{k}}\left(\vec{p}^{*} ; t\right)+\mathrm{e}^{\mathrm{i} \vec{k} \cdot \hat{\sigma} \sigma} f_{0}\left(p_{1}^{*}\right) f_{\vec{k}}\left(\vec{p}_{1}^{*} ; t\right)-\mathrm{e}^{\mathrm{i} \vec{k} \cdot \hat{\vec{\sigma} \sigma}} f_{0}(p) f_{\vec{k}}\left(\vec{p}_{1} ; t\right)\right] \\
& \quad+\mathrm{i} \Omega_{n h}(\vec{k} ; \vec{p}) h_{\vec{k}}^{\mathrm{int}}(t)-\int \mathrm{d} \vec{p}^{\prime} \int_{-\infty}^{t} \mathrm{~d} t^{\prime} \mathrm{e}^{\varepsilon\left(t-t^{\prime}\right)} \varphi_{n n}^{l}\left(\vec{k} ; \vec{p}, \vec{p}^{\prime} ; t, t^{\prime}\right) f_{\vec{k}}\left(\vec{p}^{\prime} ; t^{\prime}\right) \\
& \quad-\int_{-\infty}^{t} \mathrm{~d} t^{\prime} \mathrm{e}^{\varepsilon\left(t-t^{\prime}\right)} \varphi_{n h}\left(\vec{k} ; \vec{p} ; t, t^{\prime}\right) h_{\vec{k}}^{\mathrm{int}}\left(t^{\prime}\right)
\end{aligned}
$$

where $c_{2}^{0}(\vec{k})$ is the low-density limit of the direct correlation function. $\sigma$ is the hard-sphere diameter and the pair distribution function $g_{2}(\sigma)$. The step function $\Theta_{-}(x)$ is unity for $x<0$ and vanishes otherwise. $d \Omega_{\sigma}$ is the differential solid angle, $\hat{\vec{\sigma}}$ is unity vector. The pre- and postcollision momenta of the colliding hard spheres are denoted as $\left(\vec{p}, \vec{p}_{1}\right)$ and $\left(\vec{p}^{*}, \vec{p}_{1}^{*}\right)$, respectively. $\varphi_{n n}^{l}\left(\vec{k} ; \vec{p}, \vec{p}^{\prime} ; t, t^{\prime}\right)$ is the part of transport kernel, related to long-range interaction potential $\Phi^{l}\left(\left|\vec{r}_{i j}\right|\right)$. That, is presented equation contains the Enskog-Boltzmann collision integral describing the short-time dynamics of the hard sphere model. And the collective effects connected with the long-range interactions between particles are described by the functions $\mathrm{i}_{n h}(\vec{k} ; \vec{p}), \varphi_{n n}^{l}\left(\vec{k} ; \vec{p}, \vec{p}^{\prime} ; t, t^{\prime}\right), \varphi_{n h}\left(\vec{k} ; t, t^{\prime}\right)$ and the equation for $h_{\vec{k}}^{\text {int }}(t)$. Since the collective modes for the Enskog-Boltzmann model are well studied [25], the investigation of time correlation functions and collective modes for the system of particles interacting through the potential (74) turns out to be of great interest. 


\section{Conclusions}

In this paper in order to consistently describe the kinetic and hydrodynamic processes in dense gases and liquids, the generalized non-Markovian equations for a nonequilibrium one-particle distribution function and the averaged value of the potential part of the enthalpy density are obtained using the nonequilibrium statistical operator method. The inner structure of generalized transport kernels (memory functions) for these equations was analyzed in detail. It is shown that they are expressed in terms of time correlation functions related to the basic set of dynamical variables $\hat{n}_{\vec{k}}(\vec{p})$,

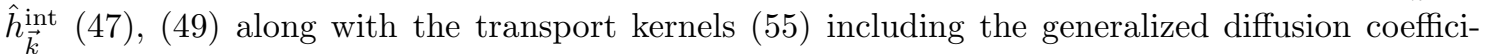
ent. Herewith, the collision integral of the kinetic equation (158) for $f_{\vec{k}}(\vec{p} ; t)$ has the Fokker-Planck structure with the generalized friction coefficient in momentum space. It also contains contributions from a generalized diffusion coefficient in momentum space as well as contributions from the dissipative hydrodynamic processes connected with the potential part of the enthalpy density. On the basis of these transport equations, a set of equations for the time correlation functions corresponding to the basic set $\hat{n}_{\vec{k}}(\vec{p}), \hat{h}_{\vec{k}}^{\text {int }}$ is obtained. Projecting it onto the first three moments of a nonequilibrium one-particle distribution function, a system of equations is obtained for the time correlation functions of hydrodynamic variables $\hat{n}_{\vec{k}}, \hat{\vec{\jmath}}_{\vec{k}}, \hat{h}_{\vec{k}}^{\text {kin }}$ and $\hat{h}_{\vec{k}}^{\text {int }}$ with separate contributions of the kinetic and potential parts of enthalpy density. Such a system in the Markovian approximation can present an interest from the point of view of investigating the dynamic structure factor, time correlation functions $\Phi_{h h}^{\text {kin,kin }}(\vec{k} ; t), \Phi_{h h}^{\text {kin,int }}(\vec{k} ; t), \Phi_{h h}^{\text {int,int }}(\vec{k} ; t)$ as well as collective modes for liquids within the generalized collective modes approach [27, 28].

\section{References}

1. John M.S., Forster D. Phys. Rev. A, 1975, 12, 254.

2. Boon J.P., Yip S. Molecular Hydrodynamics. Dover, New York, 1991.

3. Zubarev D.N., Morozov V.G. Theor. Math. Phys., 1984, 60, 814.

4. Zubarev D.N., Morozov V.G., Omelyan I.P. and Tokarchuk M.V. Theor. Math. Phys. 1991, 87, 412.

5. Klimontovich Yu.L. Kinetic theory of nonideal gas and nonideal plasma. Nauka, Moscow, 1975 (in Russian).

6. Klimontovich Yu.L. Theor. Math. Phys. 1992, 92, 312 (in Russian).

7. Zubarev D.N., Morozov V.G., Omelyan I.P., Tokarchuk M.V. Theor. Math. Phys. 1993, 96, 997.

8. Tokarchuk M.V. Theor. Math. Phys. 1993, 97, 27.

9. Tokarchuk M.V., Omelyan I.P., Kobryn A.E. Condens. Matter Phys., 1998, 1, 687.

10. Zubarev D.N., Morozov V.G., Röpke G. Statistical Mechanics of Nonequilibrium Processes:1. Basic Concepts, Kinetic Theory. Alademie Verlag, Berlin, 1996.

11. Zubarev D.N. Nonequilibrium Statistical Thermodynamics. Consultant Bureau, New-York, 1974.

12. Markiv B.B., Tokarchuk M.V. Fiz. Zbirnyk, 2008, 7, 100 (in Ukrainian).

13. Akcasu A.Z., Duderstadt J.J. Phys. Rev., 1969, 188, 479.

14. Forster D., Martin P.C. Phys. Rev. A, 1970, 2, 1575.

15. Mazenko G.F. Phys. Rev. A, 1971, 3, 2121.

16. Mazenko G.F. Phys. Rev. A, 1972, 5, 2545.

17. Mazenko G.F., Tomas Y.S., Sidney Yip. Phys. Rev. A, 1972, 5, 1981.

18. Mazenko G.F. Phys. Rev. A, 1973, 7, 209.

19. Mazenko G.F. Phys. Rev. A, 1973, 7, 222.

20. Mazenko G.F. Phys. Rev. A, 1974, 9, 360.

21. Forster D. Phys. Rev. A, 1974, 9, 943.

22. Boley C.D., Desai R.C. Phys. Rev. A, 1973, 7, 1700.

23. Furtado P.M., Mazenko G.F., Sidney Yip. Phys. Rev. A, 1975, 12, 1653.

24. Sjodin S., Sjolander A. Phys. Rev. A, 1978, 18, 1723.

25. de Schepper I.M., Cohen E.G.D. J. Stat. Phys., 1982, 27, 223.

26. Balucani U., Zoppi M. Dynamics of the Liquid State. Clarendon Press, Oxford, 1994.

27. Mryglod I.M., Omelyan I.P., Tokarchuk M.V. Mol. Phys., 1995, 84, 235.

28. Mryglod I.M. Condens. Matter Phys., 1998, 1, 753. 


\title{
До проблеми узгодженого опису кінетичних та гідродинамічних процесів у густих газах та рідинах
}

\author{
Б.Б. Марків, І.П. Омелян, М.В. Токарчук
}

Інститут фізики конденсованих систем НАН України, 79011 Львів, вул. Свєнціцького, 1

Для узгодженого опису кінетичних та гідродинамічних процесів у густих газах та рідинах отримано узагальнені немарківські рівняння для нерівноважної одночастинкової функції розподілу та середнього значення густини потенціальної частини ентальпії. Розкрито внутрішню структуру узагальнених ядер переносу даних рівнянь. Показано, що інтеграл зіткнення кінетичного рівняння має структуру Фоккера-Планка з узагальненим коефіцієнтом тертя в імпульсному просторі. Він також містить вклади від узагальненого коефіцієнта дифузії в просторі імпульсів і дисипативних процесів, пов'язаних із густиною потенціальної частини ентальпії.

Ключові слова: кінетика, гідродинаміка, кінетичні рівняння, функції пам'яті, часові кореляційні функції 